\title{
Mesoscopic simulation of three- dimensional pool boiling based on a phase-change cascaded lattice Boltzmann method
}

Cite as: Phys. Fluids 32, 103312 (2020); https://doi.org/10.1063/5.0023639

Submitted: 31 July 2020 . Accepted: 08 October 2020 . Published Online: 22 October 2020

Linlin Fei (费林林) (D), Jiapei Yang (杨家培), Yiran Chen (陈怡然), Huangrui Mo (莫晃锐), and Kai H. Luo (罗开红) (D)

\section{COLLECTIONS}

Paper published as part of the special topic on Special Issue on the Lattice Boltzmann Method
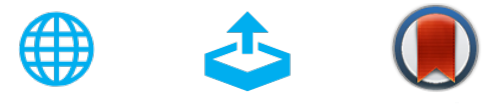

View Online

\section{ARTICLES YOU MAY BE INTERESTED IN}

A mass-conserved fractional step axisymmetric lattice Boltzmann flux solver for incompressible multiphase flows with large density ratio

Physics of Fluids 32, 103308 (2020); https://doi.org/10.1063/5.0022050

Numerical investigation of aerosol transport in a classroom with relevance to COVID-19 Physics of Fluids 32, 103311 (2020); https://doi.org/10.1063/5.0029118

Morphological and non-equilibrium analysis of coupled Rayleigh-Taylor-Kelvin-Helmholtz instability

Physics of Fluids 32, 104111 (2020); https://doi.org/10.1063/5.0023364

\section{Physics of Fluids}




\title{
Mesoscopic simulation of three-dimensional pool boiling based on a phase-change cascaded lattice Boltzmann method
}

\author{
Cite as: Phys. Fluids 32, 103312 (2020); doi: 10.1063/5.0023639 \\ Submitted: 31 July 2020 - Accepted: 8 October 2020 • \\ Published Online: 22 October 2020
}

Linlin Fei (费林林), ${ }^{1,2}$ (D) Jiapei Yang (杨家培), ${ }^{3}$ Yiran Chen (陈怡然), ${ }^{1,2,4}$ Huangrui Mo (莫晃锐),

and Kai H. Luo (罗开红) ${ }^{5, a)}$ (D)

\author{
AFFILIATIONS \\ ${ }^{1}$ Institute of Applied Physics and Computational Mathematics, Beijing 100094, China \\ ${ }^{2}$ Center for Combustion Energy, Key Laboratory for Thermal Science and Power Engineering of Ministry of Education, \\ Department of Energy and Power Engineering, Tsinghua University, Beijing 100084, China \\ ${ }^{3}$ State Key Laboratory of Automotive Safety and Energy, School of Vehicle and Mobility, Tsinghua University, \\ Beijing 100084, China \\ ${ }^{4}$ CAEP Software Center for High Performance Numerical Simulation, Beijing 100088, China \\ ${ }^{5}$ Department of Mechanical Engineering, University College London, Torrington Place, London WC1E 7JE, United Kingdom
}

Note: This paper is part of the Special Issue on the Lattice Boltzmann Method.

a) Author to whom correspondence should be addressed: K.Luo@ucl.ac.uK

\begin{abstract}
In this paper, a three-dimensional (3D) cascaded lattice Boltzmann method (CLBM) is implemented to simulate the liquid-vapor phasechange process. The multiphase flow field is solved by incorporating the pseudopotential multiphase model into an improved CLBM, the temperature field is solved by the finite difference method, and the two fields are coupled via a non-ideal equation of state. Through numerical simulations of several canonical problems, it is verified that the proposed phase-change CLBM is applicable for both the isothermal multiphase flow and the liquid-vapor phase-change process. Using the developed method, a complete 3D pool boiling process with up to hundreds of spontaneously generated bubbles is simulated, faithfully reproducing the nucleate boiling, transition boiling, and film boiling regimes. It is shown that the critical heat flux predicted by the $3 \mathrm{D}$ simulations agrees better with the established theories and correlation equations than that obtained by two-dimensional simulations. Furthermore, it is found that with the increase in the wall superheats, the bubble footprint area distribution changes from an exponential distribution to a power-law distribution, in agreement with experimental observations. In addition, insights into the instantaneous and time-averaged characteristics of the first two largest bubble footprints are obtained.
\end{abstract}

Published under license by AIP Publishing. https://doi.org/10.1063/5.0023639

\section{INTRODUCTION}

Boiling is a very efficient heat transfer process, which is commonly observed in our daily life and widely used in many industrial applications, ranging from energy conversion in nuclear reactors to cooling of microelectronic devices. Since Nukiyama's pioneering work on the classification of boiling regimes, ${ }^{1}$ research on boiling phenomena has attracted a lot of attention in the past few decades. ${ }^{1-3}$ In the boiling process, violent vaporization usually occurs simultaneously inside the fluid and on the superheated surface. With the increase in the wall superheat, three boiling regimes can be identified: ${ }^{1,2}$ nucleate boiling, transition boiling, and film boiling. For a boiling system, pool boiling means that the vapor bubbles and heated liquid are only driven by the buoyancy force, while forced-convection boiling involves other forces such as the pressure gradient driven by pumps.

Many boiling experiments have been conducted to study the bubble dynamics, as measured by the bubble departure diameter and 
bubble growth rate, and a lot of correlation equations have been consequently developed. ${ }^{4,5}$ Furthermore, extensive experiments have been carried out to investigate the boiling heat transfer characteristics, and various heat transfer models have been proposed for different boiling regimes, such as the nucleate boiling heat transfer model by Rohsenow, ${ }^{6}$ the transition boiling heat transfer model by Ohtake and Koizumi, and the critical heat flux (CHF) model by Zuber. However, most of the bubble dynamic correlations/models are limited to narrow parameter ranges (such as the pressure range), and some of them may give conflicting predictions of the bubble departure diameter and/or bubble growth rate under certain conditions, not to mention the heat transfer models.

Boiling is extremely complex that includes many coupled phenomena, such as bubble nucleation, growth, departure, deformation and coalescence, the transfer of the phase-change latent heat, and the liquid-vapor interface instability. ${ }^{9}$ With the rapid development of computer technology, numerical simulations are playing a more and more important role in elucidating the mechanisms behind the boiling phenomena. The pioneering attempt of numerical investigation for the film boiling was made by Son and Dhir based on a levelset method. ${ }^{10}$ By adding a source term to the continuity equation, the front-tracking method was extended to simulate the film boiling. ${ }^{11}$ In addition, Welch and Wilson developed a volume-of-fluid method to simulate the horizontal film boiling in $2000 .{ }^{12}$ Since then, a lot of macroscopic methods have been proposed and employed to investigate boiling phenomena. For details, the interested readers are kindly directed to the comprehensive reviews. ${ }^{13,14}$ It may be noted that most of these macroscopic methods assume an initial vapor phase as an artificial input. Therefore, these methods are limited to the film boiling regime and cannot simulate the bubble nucleation in the boiling process.

During the last three decades, the mesoscopic lattice Boltzmann method (LBM), based on the kinetic theory, has become an increasingly important method for numerical simulations of multiphase flows, on account of its generality, easy implementation, and computational efficiency. ${ }^{15-29}$ Among others, the pseudopotential model has attracted significant attention in the modeling and simulation of the liquid-vapor phase-change processes. In the pseudopotential model, the interactions among populations of molecules are modeled by a density-dependent pseudopotential. The most distinct feature of the pseudopotential model is that the phase separation and interfaces between different phases emerge naturally via the pseudopotential based interaction force among the neighboring fluid particles. ${ }^{18,30}$ As a result, deformation, movement, breakdown, and merging of phase interfaces occur automatically, without resorting to any specific techniques to track or capture interfaces, ${ }^{3}$ as usually required in traditional numerical methods. ${ }^{32}$ In 2003, the pseudopotential model was extended to simulate the boiling process by Zhang and Chen. ${ }^{33}$ In their model, the pressure difference between the real pressure $p_{E O S}$, which is based on the equation of state (EOS) with variable temperature, and the pressure in the standard isothermal LBM is incorporated via a pseudopotential based interaction force. Besides, an extended Lax-Wendroff scheme is adopted to solve the scalar energy transport equation. Since then, Hazi and Márkus, ${ }^{34}$ Biferale et al., ${ }^{35}$ and Gong and Cheng ${ }^{36}$ have proposed different liquid-vapor phase-change LBMs and used their models to simulate the boiling process. A common feature of these phase-change $\mathrm{LBMs}^{34-36}$ is that all of them are based on the double-distribution-function (DDF) method. That is to say, one lattice Boltzmann equation is used to simulate the multiphase flow field, while another lattice Boltzmann equation is used to solve the scalar temperature or energy transport equation. According to the analysis by $\mathrm{Li}$ and Luo, ${ }^{37}$ for the thermal LBMs constructed within the DDF framework, the forcing terms will introduce an additional term into the temperature transport equation. In many cases, the effect of such an additional term is negligible. However, for the thermal multiphase flow (such as the case of boiling), due to the large density change near the liquid-vapor interface, the additional term will lead to significant numerical errors in the simulation results. ${ }^{37}$ Considering this factor, Li et al. ${ }^{30}$ proposed a new hybrid phasechange LBM, in which the lattice Boltzmann method is adopted to solve the flow field, the finite difference scheme is used to solve the temperature field, and the coupling between the two fields is established by a non-ideal equation of state (EOS). Using their model, the boiling curve including the three classical boiling stages was successfully reproduced, and the effects of the heating surface wettability on the boiling heat transfer were also investigated. ${ }^{30}$ Combined with a theoretical analysis, Li et al. further clarified the mechanism of nucleation in LB simulations, which is attributed to the decrease in the fluid density near the heating surfaces, such that the system enters the unstable region of the liquid-vapor coexistence phase diagram.

In the classical collision-streaming algorithm of the LBM, a widely used collision operator is the single-relaxation-time (SRT) operator, in which all the distribution functions (DFs) are relaxed to their local equilibria with an identical time scale. ${ }^{39}$ However, the SRT-LBM may suffer from instability under a high Reynolds number or low-viscosity conditions. ${ }^{40,41}$ The multiple-relaxationtime (MRT) operator is an improved collision operator, in which the collision is carried out in the raw moment space, and different moments of DFs can be relaxed separately. In 2006, a cascaded operator was proposed by Geier et al. ${ }^{42}$ In the cascaded operator, the collision is executed in the central moment space; thus, it is also interpreted as a "central-moments-based" operator. Compared with the SRT-LBM, both the MRT-LBM and the cascaded LBM (CLBM) can enhance numerical stability by carefully separating the time scales among the kinetic modes. ${ }^{42,43}$ Studies also showed that the cascaded operator outperforms the MRT operator in terms of numerical stability and Galilean invariance. ${ }^{21,42,43}$ More recently, the relation between the CLBM and the MRT-LBM has been clarified to form a generalized MRT (GRMT) framework. ${ }^{44,45}$ Within the GMRT framework, the inconsistencies in terms of forcing schemes and/or equilibrium central moments in some previous CLBMs ${ }^{43,46,47}$ have also been revised. ${ }^{44,45}$ So far, CLBM has been well verified and obtained great success in the modeling of both the isothermal multiphase flow ${ }^{17,21,43}$ and the single-phase thermal flow. ${ }^{48,49}$ Therefore, it is time to extend CLBM to simulate the more challenging thermal multiphase flow, e.g., the boiling process. More recently, CLBM has been employed to simulate twodimensional (2D) forced-convection boiling on a cylinder by Saito et al., ${ }^{50}$ while the present work further extends CLBM to a much larger thermal multiphase system, i.e., the three-dimensional (3D) pool boiling.

This paper proposes a 3D CLBM for multiphase flows with phase-change process based on the pseudopotential model and then uses it to investigate the pool boiling process. The rest of this paper is 
structured as follows: In Sec. II, the proposed phase-change CLBM is described in detail. Numerical experiments are carried out for several benchmark problems to validate the proposed method in Sec. III. Section IV presents numerical simulations of the 3D pool boiling process, as well as analyses and discussions of results. Finally, concluding remarks are given in Sec. $V$.

\section{CLBM FOR THE LIQUID-VAPOR PHASE-CHANGE PROCESS}

In this section, a 3D hybrid CLBM for the liquid-vapor phasechange process is proposed, in which the flow field is solved by an improved CLBM coupled with the pseudopotential model, the temperature field is solved by the finite difference method, and the two fields are coupled via the non-ideal equation of state.

\section{A. Solution of the flow field}

To construct the CLBM for the 3D flow field, the D3Q19 lattice is employed, in which the continuous velocity space is represented by a discrete velocity set, $\mathbf{e}_{i}=\left[\left|e_{i x}\right\rangle,\left|e_{i y}\right\rangle,\left|e_{i z}\right\rangle\right]$, with 19 discrete velocities defined as follows: ${ }^{3}$

$$
\begin{aligned}
& \left|e_{i x}\right\rangle=[0,1,-1,0,0,0,0,1,-1,1,-1,1,-1,1,-1,0,0,0,0]^{\top}, \\
& \left|e_{i y}\right\rangle=[0,0,0,1,-1,0,0,1,1,-1,-1,0,0,0,0,1,-1,1,-1]^{\top}, \\
& \left|e_{i z}\right\rangle=[0,0,0,0,0,1,-1,0,0,0,0,1,1,-1,-1,1,1,-1,-1]^{\top},
\end{aligned}
$$

where $i=0,1, \ldots, 18$ and $|\cdot\rangle$ denotes a nineteen-dimensional column vector. The lattice speed $c=\delta x / \delta t=1$ and lattice sound speed $c_{s}=1 / \sqrt{3}$ are adopted, where $\delta x=1$ and $\delta t=1$ are the lattice spacing and time step, respectively. The raw and central moments of the discrete distribution functions (DFs) $f_{i}$ are defined as follows:

$$
\begin{gathered}
k_{m n p}=\left\langle f_{i} \mid e_{i x}^{m} e_{i y}^{n} e_{i z}^{p}\right\rangle, \\
\tilde{k}_{m n p}=\left\langle f_{i} \mid\left(e_{i x}-u_{x}\right)^{m}\left(e_{i y}-u_{y}\right)^{n}\left(e_{i z}-u_{z}\right)^{p}\right\rangle,
\end{gathered}
$$

where $m, n$, and $p$ are integers and $u_{x}, u_{y}$, and $u_{z}$ are velocity components in the $x, y$, and $z$ directions, respectively. The equilibrium raw and central moments, $k_{m n p}^{e q}$ and $\tilde{k}_{m n p}^{e q}$, are defined, likewise, by replac$\operatorname{ing} f_{i}$ with the discrete equilibrium distribution functions (EDFs) $f_{i}^{e q}$. To construct the central-moments-based collision operator, a raw moment set $\left|T_{i}\right\rangle$ and the corresponding central moment set $\left|\tilde{T}_{i}\right\rangle$ are needed,

$$
\left|T_{i}\right\rangle=\left[T_{0}, T_{1}, \ldots, T_{18}\right]^{\top},\left|\tilde{T}_{i}\right\rangle=\left[\tilde{T}_{0}, \tilde{T}_{1}, \ldots, \tilde{T}_{18}\right]^{\top},
$$

where the elements are combinations of $k_{m n p}$ and $\tilde{k}_{m n p}$ in the ascending order of $(m+n+p)$, respectively. As discussed in Ref. 45, to improve the implementation of the 3D CLBM, the following non-orthogonal central moment set is adopted in this work:

$$
\begin{gathered}
\left|\tilde{T}_{i}\right\rangle=\left[\tilde{k}_{000}, \tilde{k}_{100}, \tilde{k}_{010}, \tilde{k}_{001}, \tilde{k}_{110}, \tilde{k}_{101}, \tilde{k}_{011}, \tilde{k}_{200}, \tilde{k}_{020}, \tilde{k}_{002}, \tilde{k}_{120},\right. \\
\left.\tilde{k}_{102}, \tilde{k}_{210}, \tilde{k}_{201}, \tilde{k}_{012}, \tilde{k}_{021}, \tilde{k}_{220}, \tilde{k}_{202}, \tilde{k}_{022}\right]^{\top} .
\end{gathered}
$$

It may be noted that "non-orthogonal" means the basis vectors for the above moments are linearly independent but not necessarily orthogonal to one another. ${ }^{23}$ The corresponding raw moment set $\left|T_{i}\right\rangle$ can be defined analogously.

According to Eq. (2), the transformation from the discrete velocity space to the raw moment space can be performed via a transformation matrix $\mathbf{M}$, and the shift between the raw moment space and the central moment space can be achieved by a shift matrix $\mathbf{N}$, i.e., ${ }^{44,45}$

$$
\left|T_{i}\right\rangle=\mathbf{M}\left|f_{i}\right\rangle, \quad\left|\tilde{T}_{i}\right\rangle=\mathbf{N}\left|T_{i}\right\rangle=\mathbf{N M}\left|f_{i}\right\rangle .
$$

The explicit formulations for $\mathbf{M}$ and $\mathbf{N}$, as well as their inverses, can be obtained according to the definition in Eqs. (4) and (5) (see Appendix A). The row vector in $\mathbf{M}$ corresponds to the basis vector for each element in $\left|T_{i}\right\rangle$, with $\mathbf{M}$ being a non-orthogonal matrix. Such a non-orthogonal matrix $\mathbf{M}$ is also a sparse matrix, which has less non-zero elements compared with its orthogonal counterpart. ${ }^{23}$ Moreover, the explicit formulation of $\mathbf{N}$ is very concise because no mixed terms (such as $\tilde{k}_{200}-\tilde{k}_{020}$ ) are encountered in Eq. (4). The combined two factors, i.e., the adoptions of the non-orthogonal central moment set in Eq. (4) and the linear transformations in Eq. (5), would significantly simply the implementation and greatly reduce the computational cost of CLBM in 3D.

By relaxing different central moments to their equilibria separately, the post-collision central moments are given by ${ }^{44,45}$

$$
\left|\tilde{T}_{i}^{*}\right\rangle=\left|\tilde{T}_{i}\right\rangle-\mathbf{S}\left(\left|\tilde{T}_{i}\right\rangle-\left|\tilde{T}_{i}^{e q}\right\rangle\right)+(\mathbf{I}-\mathbf{S} / 2) \delta t\left|C_{i}\right\rangle,
$$

where $\left|C_{i}\right\rangle$ are the forcing terms in the central moment space, by which the force field $\mathbf{F}$ is incorporated into the CLBM. The relaxation matrix $\mathbf{S}$ is block-diagonal,

$$
\mathbf{S}=\operatorname{diag}\left\{s_{0}, s_{1}, s_{1}, s_{1}, s_{v}, s_{v}, s_{v},\left[\begin{array}{c}
s_{+}, s_{-}, s_{-} \\
s_{-}, s_{+}, s_{-} \\
s_{-}, s_{-}, s_{+}
\end{array}\right], s_{3}, s_{3}, s_{3}, s_{3}, s_{3}, s_{3}, s_{4}, s_{4}, s_{4}\right\}
$$

where $s_{+}=\left(s_{2 b}+2 s_{2}\right) / 3$ and $s_{-}=\left(s_{2 b}-s_{2}\right) / 3$. The $3 \times 3$ block in $\mathbf{S}$ is designed to independently relax the normal stress differences $\left(\tilde{k}_{200}-\tilde{k}_{020}\right.$ and $\left.\tilde{k}_{200}-\tilde{k}_{002}\right)$ and the trace of the pressure tensor $\left(\tilde{k}_{200}\right.$ $\left.+\tilde{k}_{020}+\tilde{k}_{002}\right)$, with the relaxation rates $s_{2 b}$ and $s_{2}$, respectively. ${ }^{45}$

According to the previous analysis, ${ }^{42,44,47}$ the central moments of $f_{i}^{e q}$ should be set equal to the continuous central moments of the Maxwell-Boltzmann distribution. Therefore, the equilibrium central moments based on the central moment set in Eq. (4) are given as follows: ${ }^{45}$

$$
\left|\tilde{T}_{i}^{e q}\right\rangle=\left[\rho, 0,0,0,0,0,0, \rho c_{s}^{2}, \rho c_{s}^{2}, \rho c_{s}^{2}, 0,0,0,0,0,0, \rho c_{s}^{4}, \rho c_{s}^{4}, \rho c_{s}^{4}\right]^{\top} .
$$

It can be proved that such a definition of $\left|\tilde{T}_{i}^{e q}\right\rangle$ is equivalent to choos$\operatorname{ing} f_{i}^{e q}$ with all Hermite polynomial tensors supported by the D3Q19 lattice, although the explicit formulation of $f_{i}^{e q}$ is usually not needed in the simulations. Consistently, the forcing terms in the central moment space are given as follows: ${ }^{45}$

$$
\begin{aligned}
\left|C_{i}\right\rangle=\left[0, F_{x}, F_{y}, F_{z}, 0,0,0,0,0,0, F_{x} c_{s}^{2}, F_{x} c_{s}^{2}, F_{y} c_{s}^{2}, F_{z} c_{s}^{2},\right. & \\
& \left.F_{y} c_{s}^{2}, F_{z} c_{s}^{2}, 0,0,0\right]^{\top} .
\end{aligned}
$$

After the collision step, the post-collision DFs are reconstructed by $\left|f_{i}^{*}\right\rangle=\mathbf{M}^{-1} \mathbf{N}^{-1}\left|\tilde{T}_{i}^{*}\right\rangle$. Then, the streaming step is performed in the discrete velocity space as usual, i.e., from point $\mathbf{x}$ to the neighbors $\left(\mathbf{x}+\mathbf{e}_{i} \delta t\right)$

$$
f_{i}\left(\mathbf{x}+\mathbf{e}_{i} \delta t, t+\delta t\right)=f_{i}^{*}(\mathbf{x}, t)
$$

The hydrodynamic variables are updated by

$$
\rho=\sum_{i} f_{i}, \quad \rho \mathbf{u}=\sum_{i} f_{i} \mathbf{e}_{i}+\delta t \mathbf{F} / 2 .
$$


The kinematic viscosity $v$ and bulk viscosity $\xi$ are related to the relaxation parameters by $v=\left(1 / s_{2}-0.5\right) c_{s}^{2} \delta t$ and $\xi=2 / 3\left(1 / s_{2 b}-0.5\right) c_{s}^{2} \delta t$, respectively.

The combination of Eqs. (6)-(11) forms our CLBM for modeling the single-phase isothermal flow. To model the phase-change process, the pseudopotential model is employed in this work, in which the interaction force among fluid particles is defined as ${ }^{15,51}$

$$
\mathbf{F}_{\text {int }}=-G \psi(\mathbf{x}) \sum_{i} w\left(\left|\mathbf{e}_{i}\right|^{2}\right) \psi\left(\mathbf{x}+\mathbf{e}_{i} \delta t, t\right) \mathbf{e}_{i},
$$

where $G$ controls the strength of the interaction force and $\psi$ is a density-based pseudopotential function. The weights are given as $w(0)=1, w(1)=1 / 6$, and $w(2)=1 / 12$. To incorporate the realistic equation of state, the pseudopotential function can be chosen as ${ }^{52}$

$$
\psi=\sqrt{2\left(p_{E O S}-\rho c_{s}^{2}\right) / G c^{2}},
$$

where $G=-1$ is used to guarantee that the term inside the square root is positive. However, the mechanical stability solution of the coexistence densities will be inconsistent with the solution given by the Maxwell construction (the so-called thermodynamic inconsistency of the pseudopotential model) if such a square-root-form pseudopotential is used. To solve this problem, Li et al. suggested that the mechanical stability condition could be adjusted to achieve the thermodynamic consistency by modifying the forcing terms for both SRT-LBM ${ }^{53}$ and MRT-LBM. ${ }^{54}$ Following the methodology of Li et al., the forcing terms in the present CLBM could be modified as

$$
\begin{aligned}
\left|C_{i}\right\rangle=\left[0, F_{x}, F_{y}, F_{z}, 0,0,0, \eta, \eta, \eta, F_{x} c_{s}^{2}, F_{x} c_{s}^{2}, F_{y} c_{s}^{2}, F_{z} c_{s}^{2},\right. \\
\left.F_{y} c_{s}^{2}, F_{z} c_{s}^{2}, 0,0,0\right]^{\top},
\end{aligned}
$$

where $\eta$ is defined as

$$
\eta=\frac{2 \sigma\left|\mathbf{F}_{\mathrm{int}}\right|^{2}}{\psi^{2}\left(s_{e}^{-1}-0.5\right) \delta t},
$$

in which $\sigma$ is used to adjust the mechanical stability condition, within the tunable range, $0.0625 \leq \sigma \leq 0.125$. It should be noted that only the interaction force $\mathbf{F}_{\text {int }}$ (instead of the total force $\mathbf{F}$ ) is needed in the adjustment term $\eta$.

Using the Chapman-Enskog analysis, the following macroscopic equations can be recovered in the low-Mach number limit:

$$
\begin{aligned}
\partial_{t} \rho+\nabla \cdot(\rho \mathbf{u})= & 0 \\
\partial_{t}(\rho \mathbf{u})+\nabla \cdot(\rho \mathbf{u u})= & -\nabla\left(\rho c_{s}^{2}\right)+\nabla \cdot\left[\rho v\left(\nabla \mathbf{u}+(\nabla \mathbf{u})^{\mathrm{T}}\right)\right. \\
& \left.-\frac{2}{3} \rho v(\nabla \cdot \mathbf{u}) \mathbf{I}\right]+\nabla\left[\rho v_{b}(\nabla \cdot \mathbf{u})\right] \\
& +\mathbf{F}-2 G^{2} c^{4} \sigma \nabla \cdot\left(|\nabla \psi|^{2} \mathbf{I}\right)
\end{aligned}
$$

where the last term in the momentum equation is an additional term introduced by the adjustment term $\eta$. When $\sigma=0$, Eq. (14) degrades into Eq. (9), and the above equations degrade into the standard Navier-Stokes equations.

\section{B. Solution of the temperature field}

The temperature equation for the liquid-vapor phase-change process can be written as ${ }^{30}$

$$
\frac{\partial T}{\partial t}=-\mathbf{u} \cdot \nabla T+\frac{1}{\rho c_{v}}\left(\lambda \nabla^{2} T+\nabla \lambda \cdot \nabla T\right)-\frac{T}{\rho c_{v}}\left(\frac{\partial p_{\mathrm{EOS}}}{\partial T}\right)_{\rho} \nabla \cdot \mathbf{u},
$$

where $\lambda$ is the thermal conductivity and $c_{v}$ is the specific heat at constant volume. It may be noted that for convenience, the term $\nabla \cdot(\lambda \nabla T)$ used in Ref. 30 has been replaced by $\left(\lambda \nabla^{2} T+\nabla \lambda \cdot \nabla T\right)$. In this work, we use the finite difference method, originally suggested by Li et al., ${ }^{30}$ to solve the temperature field. The first-order and second-order derivatives in Eq. (17) are calculated using the following lattice-based finite difference scheme: ${ }^{5}$

$$
\begin{aligned}
\frac{\partial \phi}{\partial x_{\alpha}} & =\frac{1}{c_{s}^{2} \delta t} \sum_{i} \omega\left(\left|\mathbf{e}_{i}\right|^{2}\right) \mathrm{e}_{i \alpha} \varphi\left(\mathbf{x}+\mathbf{e}_{i} \delta t\right), \\
\frac{\partial^{2} \phi}{\partial x_{a} \partial x_{a}} & =\frac{2}{c_{s}^{2} \delta t} \sum_{i} \omega\left(\left|\mathbf{e}_{i}\right|^{2}\right)\left[\varphi\left(\mathbf{x}+\mathbf{e}_{i} \delta t\right)-\varphi(\mathbf{x})\right],
\end{aligned}
$$

where $\alpha$ represents the coordinate axis and $\phi$ represents a physical variable. Following the work of Li et al., ${ }^{30}$ if the right-hand side of Eq. (17) is denoted by $K(T)$, the time discretization is realized using the fourth-order Runge-Kutta scheme,

$$
\begin{gathered}
T^{t+\delta t}=T^{t}+\frac{\delta t}{6}\left(h_{1}+h_{2}+h_{3}+h_{4}\right), \\
h_{1}=K\left(T^{t}\right), h_{2}=K\left(T^{t}+\frac{\delta t}{2} h_{1}\right), h_{3}=K\left(T^{t}+\frac{\delta t}{2} h_{2}\right), \\
h_{4}=K\left(T^{t}+\delta t h_{3}\right) .
\end{gathered}
$$

\section{Coupling between the flow field and the temperature field}

The pseudopotential CLBM in Sec. III A and the finite difference solver in Sec. III B are coupled via the non-ideal equation of state (EOS) pEOS. Namely, the change in temperature leads to the change in $p_{E O S}$, which is, in turn, incorporated into the pseudopotential CLBM for the flow field through Eqs. (12) and (13). Different equations of state, such as the van der Waals, CarnahanStarling, and Peng-Robinson equations, can be incorporated into the pseudopotential model by the square-root-form pseudopotential in Eq. (13), in which the Peng-Robinson EOS is adopted in this work,

$$
p_{E O S}=\frac{\rho R T}{1-b \rho}-\frac{a \varphi(T) \rho^{2}}{1+2 b \rho-b^{2} \rho^{2}}
$$

where $\varphi(T)=\left[1+\left(0.37464+1.54226 \omega-0.26992 \omega^{2}\right)\left(1-\sqrt{T / T_{c}}\right)\right]^{2}$, $a=0.4572 R^{2} T_{c}^{2} / p_{c}$, and $b=0.0778 R T_{c} / p_{c}$. According to the analyses in the literature, ${ }^{52,54}$ we set $\omega=0.344, a=2 / 49, b=2 / 21$, and $R=1$. Then, the critical temperature and pressure can be obtained as $T_{c}=0.07292$ and $p_{c}=0.05957$, respectively.

In addition, in the simulations of the pool boiling, the vapor bubbles and heated liquid are driven by the buoyancy force, which is defined as

$$
\mathbf{F}_{b}=-\left(\rho-\rho_{\text {ave }}\right) g \mathbf{j}
$$


where $\rho_{\text {ave }}$ is the average density in the system, $g$ is the magnitude of the gravity acceleration, and $\mathbf{j}$ is the unit vector in the vertical direction. As a result, the total force acting on the system is $\mathbf{F}=\mathbf{F}_{\text {int }}+\mathbf{F}_{b}$.

\section{NUMERICAL VERIFICATION}

In this section, numerical simulations are conducted to assess the validity of the proposed liquid-vapor phase-change CLBM for both isothermal multiphase flow and thermal multiphase flow. Unless otherwise specified, the tunable relaxation parameters are set to be $s_{2 b}=0.8, s_{3}=1.2$, and $s_{4}=1.2$, and the non-equilibrium bounce-back boundary scheme is employed to deal with the non-slip velocity boundary condition (see Appendix B).

\section{A. Verification of the Laplace's law}

First, the Laplace's law is verified based on the static droplet test. In simulations, the saturation temperature is set to be $T_{s}=0.86 T_{c}$, and the equilibrium liquid-vapor coexistence densities can be calculated according to Eq. (20), i.e., $\rho_{l}=6.4989$ and $\rho_{v}=0.3797$. For such a choice, the tunable parameter $\sigma$ is set to be 0.102 to achieve the thermodynamic consistent coexistence densities. The kinetic viscosity is set to be uniform in the system, $v_{l}=v_{v}=0.1$, and the gravity is not considered in this case. The computational domain is a $160 \delta x \times 160 \delta x \times 160 \delta x$ box, with periodic boundary conditions along all the directions. The density field is initialized as follows:

$$
\begin{aligned}
\rho= & 0.5\left(\rho_{l}+\rho_{v}\right)-0.5\left(\rho_{l}-\rho_{v}\right) \\
& \times \tanh \left[2 \frac{\sqrt{\left(x-x_{0}\right)^{2}+\left(y-y_{0}\right)^{2}+\left(z-z_{0}\right)^{2}}-R_{0}}{W}\right],
\end{aligned}
$$

where $W=4 \delta x$ is the initial interface thickness, $R_{0}$ is the initial droplet radius, and the droplet center $\left(x_{0}, y_{0}, z_{0}\right)$ is located at the center of the computational domain. When the simulation is convergent, according to the Laplace's law, the pressure difference across the liquid-vapor interface $\Delta p$ is proportional to the interface curvature, namely,

$$
\Delta p=2 \gamma / R
$$

where $\gamma$ is the surface tension. The steady state radius $R$ is measured based on the location of the mean density $0.5\left(\rho_{l}+\rho_{v}\right)$, which may be slightly different from initial $R_{0}$. By changing the initial radius within the range $24 \delta x \leq R_{0} \leq 48 \delta x$, the surface tension can be measured according to Eq. (23). Figure 1 shows the change in $\Delta p$ with $2 / R$, and we can see a very good linear fitting, which means that the Laplace's law is well verified. Based on the slope of the fitting line, surface tension is obtained, $\gamma=0.066$.

\section{B. Measurement of the contact angle}

The wetting condition plays a very important role in the boiling heat transfer process. In this subsection, we consider to measure the contact angle between the liquid phase and the solid wall. Usually, the contact angle in the pseudopotential model can be tuned by adjusting the fluid-solid interaction force ${ }^{18}$ For simplicity, the fluid-solid interaction is not considered in this work, and liquidliquid interaction force in Eq. (12) is also not applied at the wall



FIG. 1. Numerical verification of the Laplace's law: red filled circles are the simulation results, and the solid fitting line has a slope of 0.066 .

nodes. The simulation is performed in a $200 \delta x \times 200 \delta x \times 100 \delta x$ box, with the periodic boundary condition in the $x$ and $y$ directions and the non-slip condition at $z=0$ and $z=100 \delta x$. The density field is also initialized according to Eq. (22), while the center is set at $\left(x_{0}\right.$, $\left.y_{0}, z_{0}\right)=(100,100,0)$, and the initial radius is $R_{0}=40 \delta x$. The steady density field is shown in Fig. 2 (top panel). It can be clearly seen that the droplet has deformed from the hemispherical shape to a coronal shape, which means that the wall is hydrophilic. To measure the accurate value of the contact angle, the density field at the slice $x=100 \delta x$ is obtained and shown in the bottom panel. The contact angle can be calculated from measurements of the base and height of the liquid phase in such a slice. If the base and height are denoted by $L_{1}$ and $L_{2}$, then the droplet radius can be calculated by ${ }^{56}$

$$
R=\frac{\left(4 L_{2}^{2}+L_{1}^{2}\right)}{8 L_{2}} .
$$
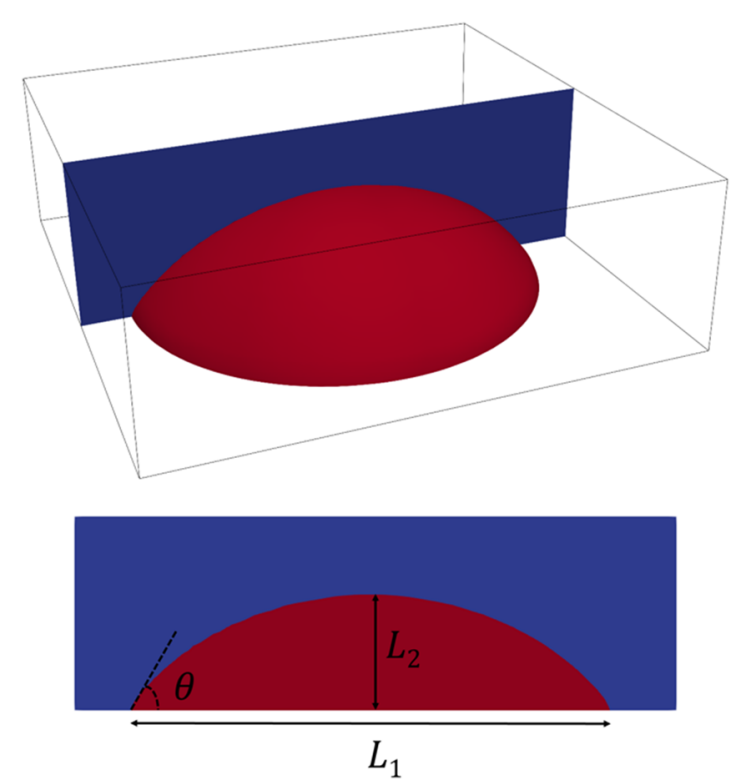

FIG. 2. Measurement of the contact angle. 
Then, the contact angle can be obtained via the formulation $\tan (\theta)=\left(L_{1} / 2\right) /\left(R-L_{2}\right)$. For the present case $\left(T_{s}=0.86 T_{c}\right)$, we finally obtain the contact angle $\theta=51.6^{\circ}$.

In the following simulations for the pool boiling process, the Jacob number (defined as the ratio between specific sensible heat and latent heat) is tuned by varying the wall temperature. Therefore, it is useful to measure the variation of the static contact angle with the temperature. As shown in Fig. 3, the contact angle increases gradually with the reduced saturation temperature and finally reaches $\theta=68.2^{\circ}$ when the temperature is approaching the critical point.

\section{Verification of the $D^{2}$ law}

In this subsection, the validity of the proposed model for simulating the liquid-vapor phase-change process is verified. To this purpose, droplet evaporation is simulated, and the results are tested based on the well-known $D^{2}$ law. ${ }^{57}$ For the single droplet evaporation case, the $D^{2}$ law predicts that the square of the droplet diameter decreases linearly with time during the evaporation process, i.e., $D^{2}(t)=D_{0}^{2}-\Omega t$, under the following conditions: (i) the evaporation process is quasi-steady, (ii) the viscous heat dissipation and the buoyancy force are negligible, and (iii) the thermophysical properties $\left(c_{p}, c_{v}\right.$, and $\left.\lambda\right)$ are constant.

We first consider the evaporation of a $3 \mathrm{D}$ liquid cylinder (equivalent to a $2 \mathrm{D}$ droplet), to verify the numerical accuracy of the present model, by comparing it with the evaporation of a $2 \mathrm{D}$ droplet by using the method of Li et al. ${ }^{30}$ The simulations are conducted in a $200 \delta x \times 200 \delta x \times \delta x$ box, with periodic boundary conditions along all the three directions. A liquid cylinder with diameter $D_{0}=70 \delta x$ is initialized in the box center. The temperature of the cylinder is set to be the saturation temperature $T_{s}=0.86 T_{c}$, and the temperature of the surrounding vapor is set to be $T_{c}$. Due to the temperature gradient at the liquid-vapor interface, evaporation is triggered. During the simulation, a constant temperature boundary condition is imposed at the boundaries in the $x$ and $y$ directions to keep the vapor temperature higher than the droplet temperature. In the computational domain, the kinetic viscosity and the specific heat at constant volume are set to be uniform, i.e., $v=0.1$ and $c_{v}=6.0$. The non-dimensional time is defined as $t^{*}=t / t_{d}$, where $t_{d}=D_{0}^{2} / v$ is the diffusion time scale. As shown in Fig. 4, the present simulations agree well with the results by $\mathrm{Li}$ et al. ${ }^{30}$ for both cases with $\lambda=1 / 3$ and $\lambda=2 / 3$, which

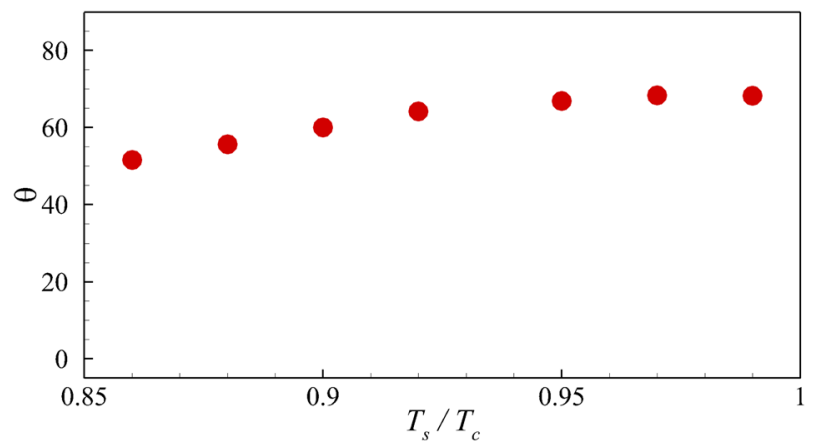

FIG. 3. Variation of the static contact angle with the reduced saturation temperature.

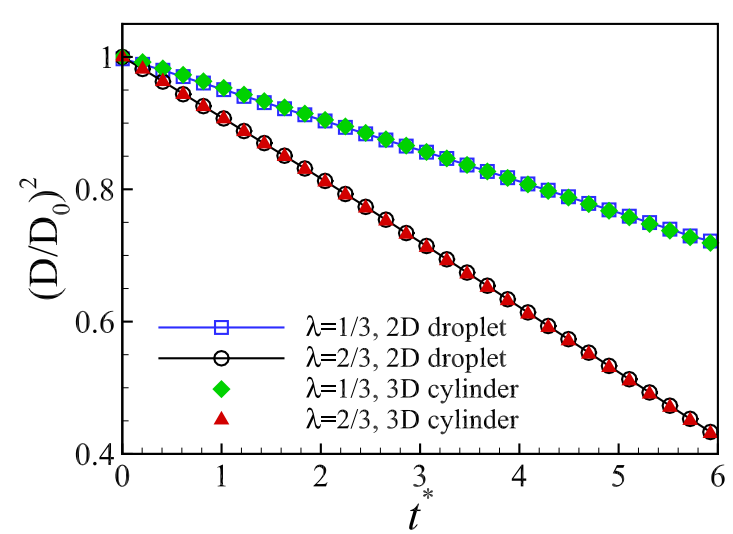

FIG. 4. Comparison of the evaporation process for a $2 \mathrm{D}$ droplet by using the method of Li et al. ${ }^{30}$ and a 3D cylinder by using the present method.

proves the accuracy of the present model for the phase-change heat transfer problems.

Then, we move to the simulation of $3 \mathrm{D}$ droplet evaporation cases in a $200 \delta x \times 200 \delta x \times 100 \delta x$ box. All the numerical settings are the same as the above, except that the constant temperature boundary condition is imposed at all the boundaries. Here, we consider three cases with $\lambda=0.125,0.25$, and 0.5 . Figure 5 shows the evaporation process for the three cases, where panel (a) is the initial state and panels (b)-(d) are the states at $t^{*}=4.81$ for $\lambda=0.125,0.25$, and 0.5 , respectively. As shown in this figure, for all cases, the spherical droplet shape is preserved during the evaporation process, and the larger the thermal conductivity is, the faster the droplet evaporates.


FIG. 5. Droplet evaporation process: (a) a snapshot of the initial droplet; (b)-(d) snapshots of the droplet at $t^{*}=4.81$ for the cases with $\lambda=0.125,0.25$, and 0.5 , respectively. 


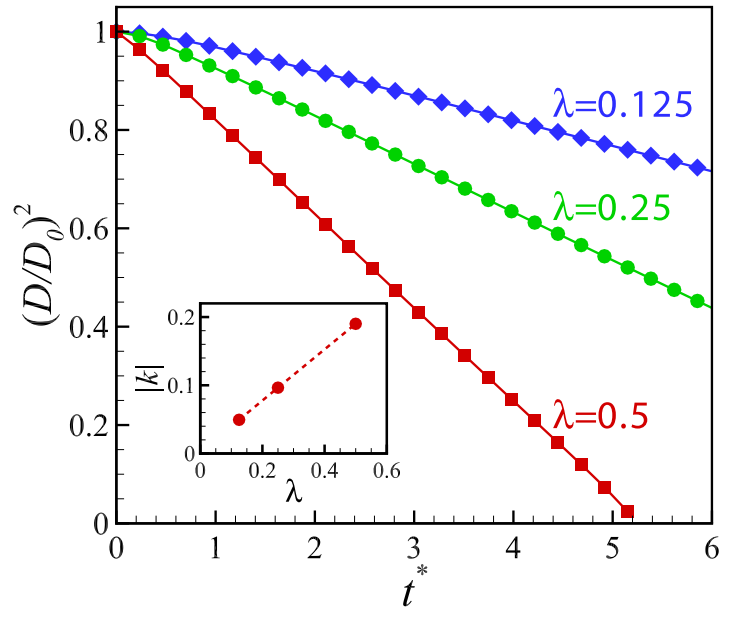

FIG. 6. The change in normalized diameter square $D^{2} / D_{0}^{2}$ with the normalized time $t^{*}$. Inset: the absolute value of the slope in the main panel $|k|$ changes linearly with $\lambda$.

To be more quantitative, the change in the normalized diameter square $D^{2} / D_{0}^{2}$ with time is plotted in Fig. 6. For all cases, $D^{2} / D_{0}^{2}$ changes linearly with $t^{*}$, which agrees well with the so-called $D^{2}$ law. Moreover, the larger the thermal conductivity is, the faster the droplet diameter square decreases with time. As can be seen in the inset of Fig. 6, the absolute value of the slope in the main panel $|k|$ changes linearly with $\lambda$, which also agrees with the theory that the evaporation constant in the $D^{2}$ law depends linearly on the thermal conductivity.

\section{NUMERICAL SIMULATIONS AND ANALYSIS}

In this section, the proposed phase-change CLBM is applied to the simulation of $3 \mathrm{D}$ pooling boiling. In the simulations, the saturation temperature is set to be $T_{s}=0.86 T_{c}$, and the tunable parameter $\sigma$ is set to be 0.102 to achieve the thermodynamic consistent coexistence densities $\left(\rho_{l}=6.4989\right.$ and $\left.\rho_{v}=0.3797\right)$. The kinetic viscosities are set to be $v_{l}=0.1$ and $v_{v}=0.5 / 3$, corresponding to the dynamic viscosity ratio $\mu_{l} / \mu_{v}=\left(\rho_{l} v_{l}\right) /\left(\rho_{v} v_{v}\right) \approx 10$. The above settings approximately correspond to the saturated water-vapor system at temperature $556 \mathrm{~K}$ and pressure $7 \mathrm{MPa} .{ }^{50}$ Following Refs. 30, 33, and 34, the specific heat at constant volume is set to be constant, $c_{v}=6.0$, in the whole computational domain. The thermal conductivity $\lambda=\rho\left(c_{v} \chi\right)$ is chosen to be proportional to the density $\rho$ with $c_{v} \chi=0.028$. $^{30}$ The specific heat at constant volume is set to be $c_{p}=c_{v}$.

The simulation is performed in a $L \times L \times H$ box, with the periodic boundary condition in the $x$ and $y$ directions and the non-slip boundary condition at the walls $z=0$ and $z=H$. The constant temperature condition is applied to the walls, with $T_{b}$ and $T_{s}\left(T_{b}>T_{s}\right)$ at the bottom wall and top wall, respectively. Following Ref. 30, we consider the hydrophilic case with a static contact angle $\theta=51.6^{\circ}$ at the saturation temperature, which corresponds to the condition with zero fluid-solid interaction force. The effect of the heating surface wettability could be investigated in the future by incorporating a fluid-solid interaction force, such as the modified $\psi$-based interaction force, ${ }^{58}$ into the present model. According to Ref. 59, characteristic length $l_{0}$, characteristic velocity $u_{0}$, and characteristic time $t_{0}$ are defined as

$$
l_{0}=\sqrt{\frac{\gamma}{g\left(\rho_{l}-\rho_{v}\right)}}, u_{0}=\sqrt{g l_{0}}, t_{0}=l_{0} / u_{0} .
$$

The liquid-vapor interface thickness measured based on the static droplet test ${ }^{23}$ is $W=3.96 \delta x$. For convenience, the characteristic physical variables used in the simulations are summarized in Table I.

It can be seen that the condition $L \gg l_{0} \gg W$ is well satisfied in the simulations, namely, the computational box length is much larger than the bubble length scale, which, in turn, is much larger than the interface thickness. Therefore, it is credible that our simulations can resolve a lot of (with up to hundreds of) bubbles. Besides, the magnitude of the maximum spurious current, measured based on the static droplet $\left(R \approx l_{0}\right)$ test, is $u_{s}=0.0018\left(\ll u_{0}\right)$. Therefore, the effect of spurious currents is negligibly small. As usual, the lattice units are used to define the above quantities. The conversion between the physical and lattice units could be found elsewhere. ${ }^{60,61}$

In the beginning of the simulation, the lower part of the computational domain $(z<0.6 H)$ is set to be the saturated liquid ( $\left.\rho=\rho_{l}, T=T_{s}\right)$, and the upper part is set to be the saturated vapor $\left(\rho=\rho_{v}, T=T_{s}\right)$. To trigger the bubble nucleation, a small temperature disturbance is added to the first grid layer near the bottom wall, i.e., $T=T_{s}+\delta T$. The disturbance term $\delta T$ satisfies the Gaussian distribution, with a mean value of 0 and a standard deviation of $0.07 T_{s}$. In the primary stage $(0 \leq t \leq 1000 \delta t)$, the gravity is not applied so that the liquid-vapor interface relaxes to a quasi-equilibrium state. A series of cases with different values of the wall superheat $\Delta T=T_{b}-T_{s}$ are considered, and the simulation runs until $t=50000 \delta t\left(t^{*}=t / t_{0} \approx 63\right)$ for each case unless otherwise specified.

For the subsequent analysis, the specific latent heat $h_{f g}$ needs to be calculated. According to the derivation by Gong and Cheng, ${ }^{62} h_{f g}$ can be obtained by

$$
h_{f g}=h_{v}-h_{l}=\int_{\rho_{v}}^{\rho_{l}} \frac{1}{\rho^{2}}\left[T\left(\frac{\partial p_{E O S}}{\partial T}\right)_{\rho}-p_{E O S}\right] d \rho+\frac{p_{E O S}}{\rho_{v}}-\frac{p_{E O S}}{\rho_{l}},
$$

where $h_{v}$ and $h_{l}$ denote the enthalpy for the vapor phase and liquid phase, respectively. In the original work by Gong and Cheng, ${ }^{62}$ the Peng-Robinson EOS is substituted into Eq. (26), and a complex formulation is obtained to calculate $h_{f g}$. In this work, the specific latent heat is obtained using standard numerical integration methods. For the Peng-Robinson EOS, we have

$$
\left(\frac{\partial p_{E O S}}{\partial T}\right)_{\rho}=\frac{\rho R}{1-b \rho}-\frac{a \rho^{2}}{1+2 b \rho-b^{2} \rho^{2}} \cdot \frac{\partial \varphi(T)}{\partial T}
$$

TABLE I. Physical variables used in the simulations (lattice units)

\begin{tabular}{lcccccc}
\hline \hline$L \times L \times H$ & $g$ & $l_{0}$ & $u_{0}$ & $u_{s}$ & $t_{0}$ & $W$ \\
\hline $600 \times 600 \times 300$ & $3 \times 10^{-5}$ & 19.02 & 0.024 & 0.0018 & 792.5 & 3.96 \\
\hline \hline
\end{tabular}




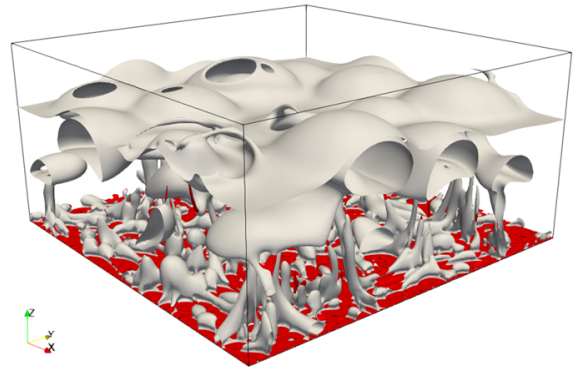

(a)

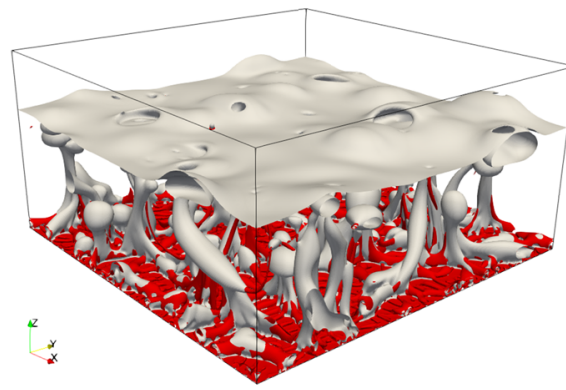

(c)

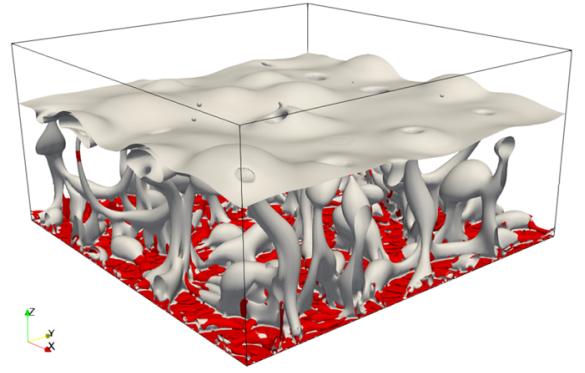

(b)

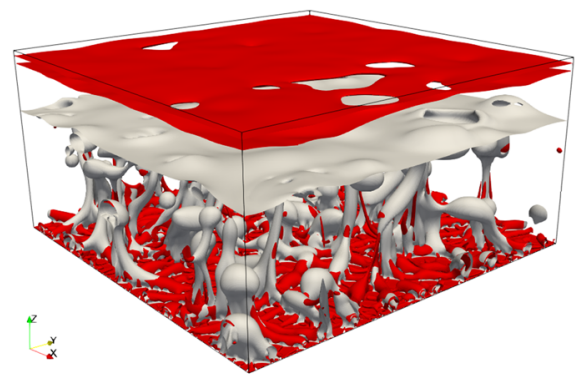

(d) where $\partial \varphi(T) / \partial T=-\left[1+\varepsilon\left(1-\sqrt{T / T_{c}}\right)\right] \varepsilon / \sqrt{T T_{c}}$ with $\varepsilon=(0.37464$ $\left.+1.54226 \omega-0.26992 \omega^{2}\right)$. Although the second term on the righthand side of Eq. (27) may be negligible, ${ }^{30}$ it is still included in our calculation. Substituting Eq. (27) into Eq. (26), the specific latent heat is obtained, $h_{f g}=0.3813$.
Figures 7-10 show the snapshots of the pool boiling process with different Jacob numbers $(\mathrm{Ja})$, where the Jacob number is defined as the ratio between specific sensible heat and latent heat, $\mathrm{Ja}$ $=c_{p} \Delta T / h_{f g}$. For the case of $J a=0.220$, it can be seen from Fig. 7(a) (at $t=20000 \delta t$ ) that due to the existence of a high-temperature zone

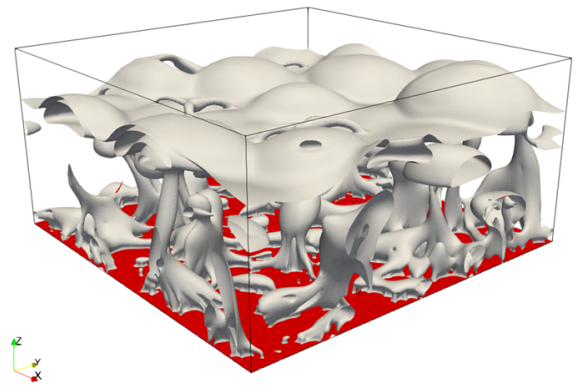

(a)

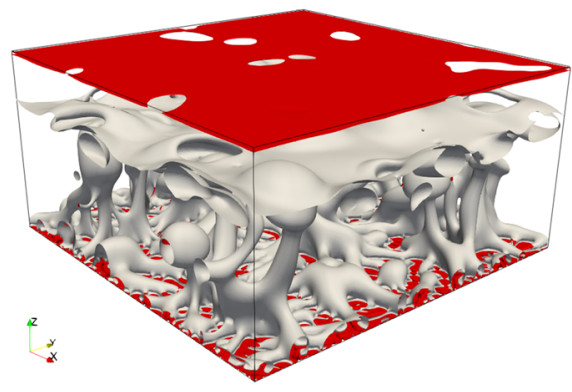

(c)



(b)

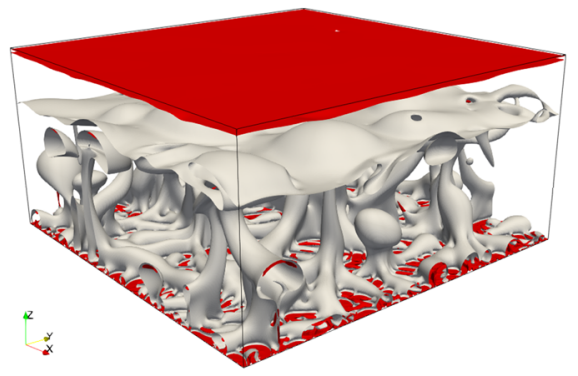

(d)
FIG. 8. Snapshots of the pool boiling process at $\mathrm{Ja}=0.283$. (a) $t^{\star}=25.24$ (b) $t^{*}=37.85$, (c) $t^{*}=50.47$, and (d) $t^{*}=63.09$. 


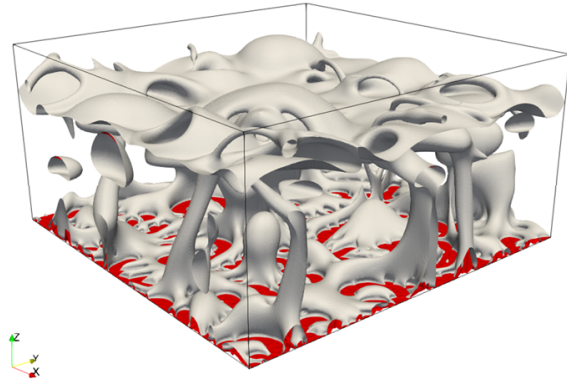

(a)

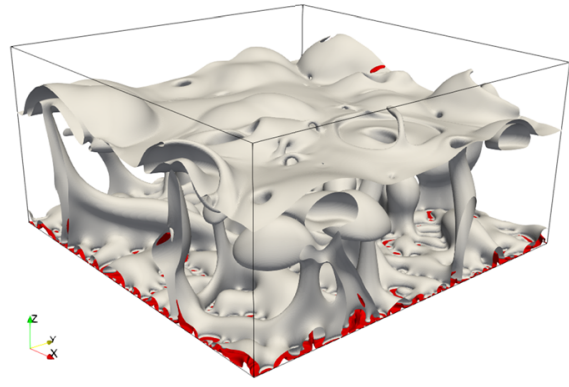

(c)

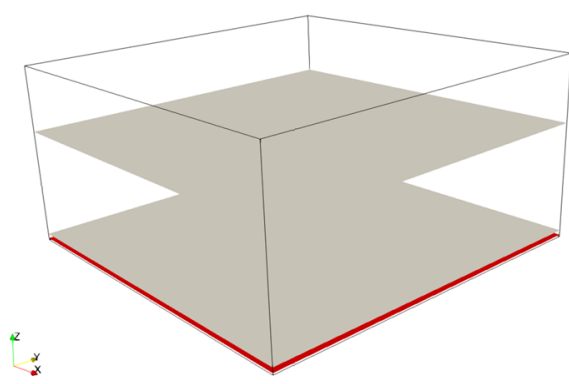

(a)

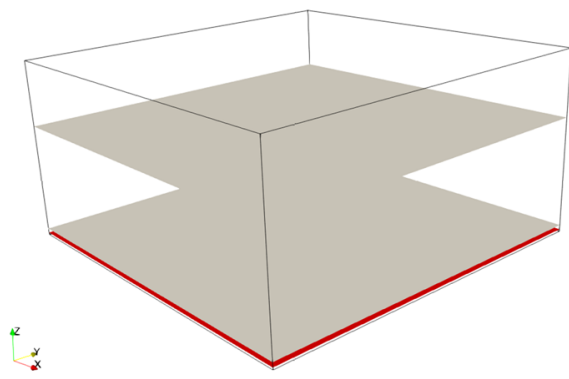

(c)

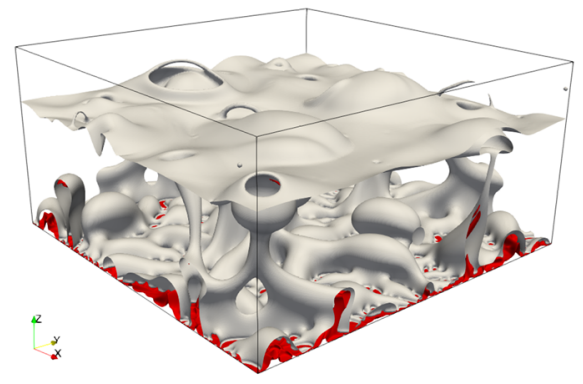

(b)



(d)



(b)

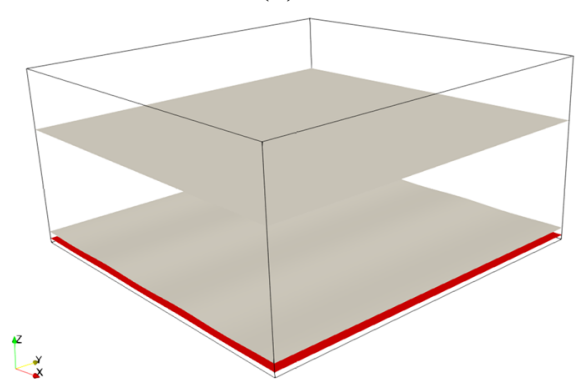

(d)
FIG. 9. Snapshots of the pool boiling process at $\mathrm{Ja}=0.441$. (a) $t^{*}=25.24$, (b) $t^{*}=37.85$, (c) $t^{*}=50.47$, and (d) $t^{*}$ $=63.09$.
FIG. 10. Snapshots of the pool boiling process at $\mathrm{Ja}=0.488$. (a) $t^{*}=25.24$, (b) $t^{*}=37.85$, (c) $t^{*}=50.47$, and (d) $t^{*}$ $=252.37$. on the wall, the liquid density is decreased according to the thermodynamic consistency, which gradually leads to the evaporation. Therefore, some small nucleation points are produced, and some of them have grown up to be small bubbles. By continuously heating, the bubbles will rise driven by the buoyancy force. When the rising bubbles reach the liquid-vapor interface, the bubbles will break, and small secondary bubbles are produced due to the interface instability, as shown in Fig. 7(b). In the meantime, the rise of bubbles leads to the rise of the high-temperature regions, which can be further confirmed by the red regions near the elongated bubble tails, as shown in Fig. 7 (c). The satellite bubbles on the wall due to the departure of rising bubbles become the new nucleation points and then 
develop to be new rising bubbles, as shown in Fig. 7(d). Moreover, as more and more bubbles grow up and carry the thermal fluids from the wall, the temperature in the main flow area will become higher and higher. Finally, a high-temperature zone can be seen above the liquid-vapor interface, as shown in Fig. 7(d). It is obvious that this case is in the nucleate boiling regime and, more specifically, in the discrete bubble region (most of the bubbles grow up and depart independently).

Then, we move to the case with a larger superheat, $J a=0.0283$, as shown in Fig. 8. Similar to Fig. 7(a), Fig. 8(a) shows the result at $t=20000 \delta t$, where some initial bubbles are going to depart. It is seen that the initial bubbles are larger than the bubbles in Fig. 7(a), which is qualitatively consistent with the positive correlation between bubble departure diameter and superheat in the classical boiling models. ${ }^{5,64}$ Larger bubbles are more likely to merge when they depart and rise, as shown in Figs. 8(b)-8(d). When the larger rising bubbles reach the liquid-vapor interface, the bubbles will also break and cause more significant disturbances. Similar to Fig. 7(d), as more and more bubbles departure and rise, the fluid in the main flow area becomes hotter and hotter, the high-temperature area also expands to be higher and higher, and, eventually, a high-temperature region will be formed above the liquid-vapor interface. Compared with the case at $J a=0.0220$, the high-temperature region reaches the upper part of the liquid-vapor interface earlier at $t^{*}=50.47$, as shown in Fig. 8(c). From different stages in Fig. 8, it is seen that the bubble departure is very efficient and is accompanied by clear coalescences among the bubbles during the boiling process. Therefore, we believe that the case at $J a=0.0283$ is in the mushroom bubble region of the nucleate boiling regime.

Figure 9 shows the results when $J a$ is increased to be 0.441 . Different from the previous cases, it is difficult to see isolated bubbles at $t=20000 \delta t\left(t^{*}=25.24\right)$, and there are clear adhesions among the tails of the rising bubbles, as shown in Fig. 9(a). Actually, most of the heating wall is covered by a vapor film for different stages in this case. Since the thermal conductivity of the vapor phase is smaller than that of the liquid phase, the vapor film is equivalent to a large thermal resistance, which works as a block between the heated wall surface and the main flow, thus reducing the heat transfer efficiency. As a result, at the moment of $t=50000 \delta t\left(t^{*}=63.09\right)$, the hightemperature region still does not expand to the main flow region substantially, not to say the area above the liquid-vapor interface. According to the results in Fig. 9, the case at $J a=0.441$ should be in the transition boiling regime.

The results at $J a=0.488$ are shown in Fig. 10. It is seen that as the superheat is further increased, the entire hot wall is covered by a continuous vapor film. The existence of the vapor film completely separates the hot wall surface from the main flow area. Under this condition, the heat transfer mechanism at the hot wall surface is only the heat conduction of the vapor phase, so the heat transfer efficiency is significantly decreased. As a result, the vapor film near the hot wall develops so slowly that its change after a long time, from $t^{*}=25.24$ [Fig. 10(a)] to $t^{*}=252.37$ [Fig. 10(d)], is still not apparent. Obviously, the system in this case is in the film boiling regime.

The above results show that with the increase in the wall superheat, the proposed phase-change CLBM can produce different boiling regimes, namely, nucleate boiling regime (discrete bubble region and mushroom bubble region), transition boiling regime, and film boiling regime. To be more quantitative, we then move to the heat transfer characteristics in the boiling process. Figure 11 shows the transient heat flux at three different $J a$, where the heat flux is defined as

$$
q=\frac{1}{L^{2}} \int_{0}^{L} \int_{0}^{L}\left[-\left.\lambda(\partial T / \partial z)\right|_{z=0}\right] d x d y
$$

For all cases, the transient heat flux has some oscillations at the initial stage $\left(t^{*}<20\right)$ and, then, gradually tends to a steady value. For the case at $J a=0.488$, its heat flux is obviously lower than the other two cases because the heat transfer efficiency is very low in the film boiling regime. In the $2 \mathrm{D}$ simulations in Ref. 30, it was shown that the heat flux fluctuates more significantly in the transition boiling regime than in the nucleate boiling regime, while in the present 3D simulations, the fluctuations in both the two boiling regimes are not too severe, which may be attributed to the $3 \mathrm{D}$ spatial average.

In order to show the heat transfer characteristics more clearly, the change in average heat flux $\bar{q}$ (average in time) with the normalized superheat (Jacob number $J a$ ), i.e., the boiling curve, is plotted in Fig. 12(a). As can be seen from Fig. 12(a), with the increase in $J a$, the average heat flux increases rapidly until a critical point (critical heat flux, CHF) is achieved. Before the CHF point, the system is in the nucleate boiling regime, including the discrete bubble region (see in Fig. 7) and the mushroom boiling region (see in Fig. 8). Then, with the further increase in superheat, the heat flux begins to decline slowly, corresponding to the transition boiling regime, and finally decreases rapidly to a very low plateau when the system enters the film boiling regime. The simulated boiling curve is in line with the classical boiling process reported in the literature. ${ }^{30,66}$ In addition, we also plot the boiling curve in the 2D limit, which is obtained by setting the computational domain as $600 \delta x \times \delta x \times 300 \delta x$. It can be seen that the $2 \mathrm{D}$ simulation results are consistent with the $3 \mathrm{D}$ results at small or large superheat but are clearly smaller than the $3 \mathrm{D}$ results in the middle region $(0.25 \leq J a \leq 0.45)$.

To explain the difference, let us refer to the classical CHF models for the pool boiling process. According to the models

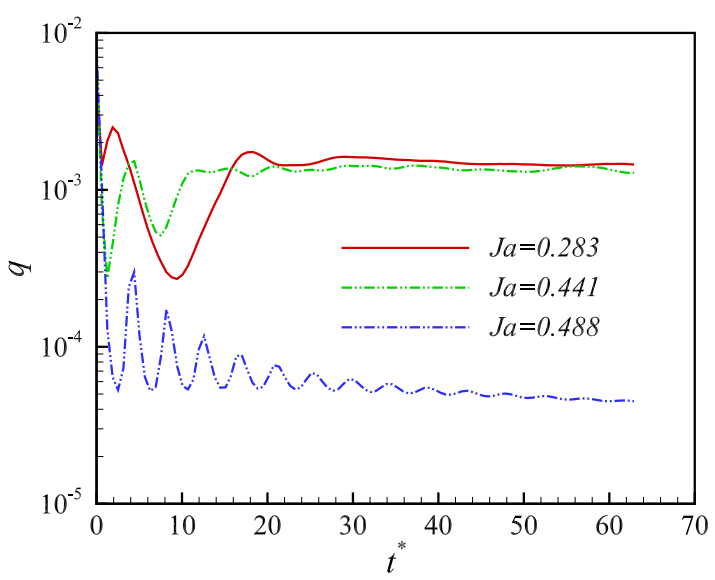

FIG. 11. Transient heat at $\mathrm{Ja}=0.283,0.441$, and 0.488 . 

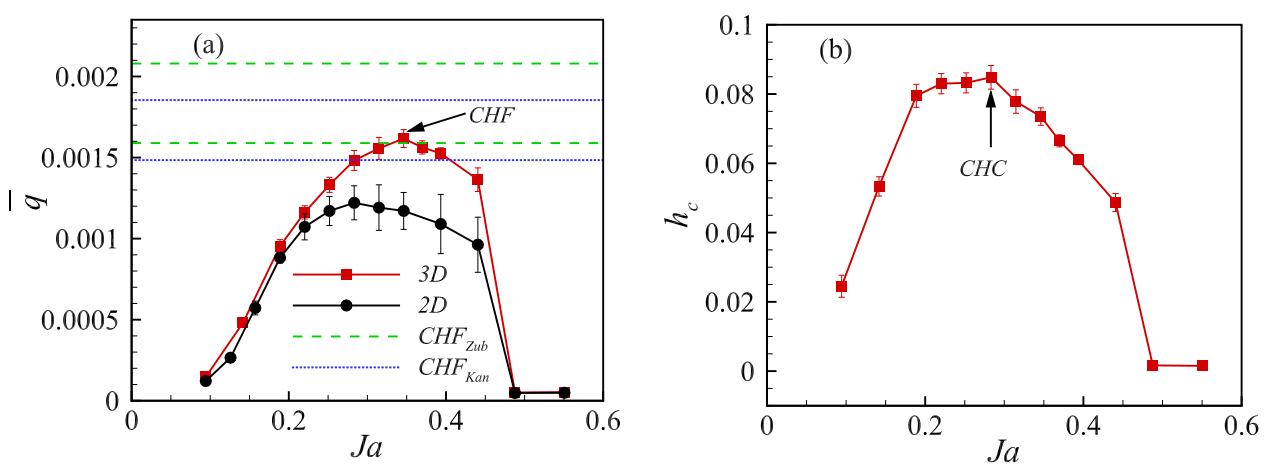

FIG. 12. (a) The change in average heat flux with Ja. The two green dashed lines (blue dotted lines) represent the upper bound (lower bound) of the CHF value predicted by using the Zuber model ${ }^{8}$ (Kandlikar mode $\left.\right|^{65}$ ). (b) The change in the heat transfer coefficient with Ja. proposed by Zuber $^{8}$ and Kandlikar, ${ }^{65}$ the CHF has the following formulation:

$$
C H F=\alpha \rho_{v} h_{f g}\left[\frac{\gamma\left(\rho_{l}-\rho_{v}\right) g}{\rho_{v}^{2}}\right]^{0.25}
$$

where the prefactor $\alpha \in[0.120,0.157]$ for the Zuber model. ${ }^{8}$ For the Kandlikar model, ${ }^{65} \alpha=\frac{1+\cos \theta}{16} \cdot\left[\frac{2}{\pi}+\frac{\pi}{4}(1+\cos \theta)\right]^{0.5}$, which is 0.140 with the contact angle $\theta=51.6^{\circ}$ at the saturation temperature. According to Fig. 3, the contact angle is not expected to be constant when the wall temperature is changed. Within the range of $J a$, the contact angle would fall within $\in\left[51.6^{\circ}, 68.2^{\circ}\right]$, and therefore, we have $\alpha \in[0.112,0.140]$ based on the Kandlikar model. The two dashed (dotted) lines in Fig. 12(a) represent the upper bound and lower bound of CHF predicted by using the Zuber model (Kandlikar model), respectively. As can be seen, the CHF by the 3D simulations locates within the predicted ranges for both models, while the CHF by the 2D simulations deviates significantly from them. Quantitatively, the deviation of CHF by $2 \mathrm{D}$ simulations from the lower bound of the Zuber model $(\alpha=0.12)$ is $(25 \pm 7) \%$, which agrees well with the $2 \mathrm{D}$ simulations by Gong and Cheng ${ }^{66}$ (although the physical properties used in this work are different from those in the work of Gong et al.).

As is known, boiling is a typical $3 \mathrm{D}$ phenomenon, where the heat flux is directly affected by the bubble dynamics, such as the bubble departure diameter and departure frequency, while the bubble dynamics are determined by the $3 \mathrm{D}$ force balance. ${ }^{4,5}$ Therefore, it is intuitively credible that the $2 \mathrm{D}$ simulation results have some deviations from the $3 \mathrm{D}$ simulation results, especially in the area with high heat transfer efficiency.

The change in the heat transfer coefficient $h_{c}=\bar{q} / \delta T$ with superheat is plotted in Fig. 12(b). The heat transfer coefficient is a measurement of the heat transfer efficiency in the boiling system, and the critical heat-transfer coefficient $(\mathrm{CHC})$ point represents the point with the highest heat transfer efficiency in practice. Comparing Figs. 12(a) and 12(b), we observe an important feature in the boiling process, namely, the $\mathrm{CHC}$ point lies on the left-hand side of the $\mathrm{CHF}$ point $\left(J a_{\mathrm{CHC}}<J a_{\mathrm{CHF}}\right)$.

As far as the authors know, this paper produces the boiling curve together with three typical boiling regimes for the large scale $3 \mathrm{D}$ pool boiling process (with hundreds of bubbles of different sizes spontaneously generated from the wall) in the lattice Boltzmann community for the first time. In the industrial applications, it is a key in modeling the bubble dynamics (departure diameter and departure frequency) for the boiling system because it is the starting point of predicting the heat flux and heat transfer coefficient. Based on the large scale $3 \mathrm{D}$ simulations in this work, it is possible to investigate the bubble footprints on the wall. Figure 13(a) shows the sketch to obtain the bubble footprint in our simulations, namely, the bubble footprints can be obtained based on the slice near the wall $(z=10 \delta x)$ in a time series. Figures $13(\mathrm{~b})-13(\mathrm{~d})$ show the typical bubble footprint distributions at $J a=0.220,0.0283$, and 0.441 , respectively. In these slices, the bubble footprints are marked in blue, and the gray background represents the liquid phase. For the subsequent analysis, the first two largest bubbles in each slice are denoted as "first" and "second," respectively. From these figures, it can be seen that with the increase in $\mathrm{Ja}$, the bubble footprints and the total area of the vapor phase become larger and larger. For the small $\mathrm{Ja}$ in Fig. 13(a), the boundary for each bubble footprint is clearly visible, and the contour is approximately circular, which is corresponding to the discrete bubble region, in agreement with the previous discussion. When $\mathrm{Ja}$ is increased to be 0.0283 , some bubbles may merge to form a large vapor spot, which is corresponding to the mushroom bubble region. When $J a$ is further increased, the system enters into the transition boiling regime, and more bubbles merge to form larger vapor spots, as shown in Fig. 13(d).

Based on the method in Fig. 13, the probability density functions (PDFs) of the bubble footprint area can be obtained, as shown in Fig. 14. For the small superheat cases $(J a=0.189$ and $J a=0.220)$, the system is in the discrete bubble region, and most of the bubbles are isolated. It is seen from Figs. 14(a) and 14(b) that the PDFs of the bubble footprint area are approximately exponentially damped and can be well fitted by

$$
P(S)=\beta_{1} \operatorname{cexp}(-\mathrm{cS}) \text {, }
$$

where $S$ is the footprint area and $c=1 /\left(\sum_{i=1}^{N} S_{i} / N\right)$ is the reciprocal of the average footprint area. Considering that the adopted multiphase LBM is a diffused interface method, only the bubble footprints with a larger length scale than the interface width $(\sqrt{S}<W)$ are included in the calculation, and the $x$ coordinate starts from $10\left(\delta x^{2}\right)$ in Fig. 14. Therefore, a normalization factor $\beta_{1}$ is introduced in Eq. (30), which is defined as $\beta_{1}=1 /\left(\int_{10}^{L^{2}} \operatorname{cexp}(-\mathrm{cS}) d \mathrm{~S}\right)$. The results confirm the validity of the recent suggestion motivated by the experiments; ${ }^{3,67}$ namely, for low heat fluxes, the PDF of the bubble footprint area obeys the exponential distribution. As the superheat is 


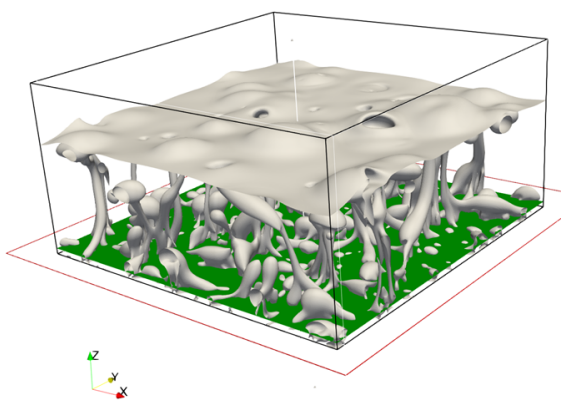

(a)

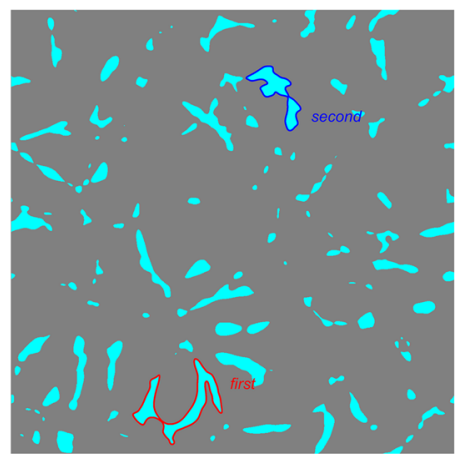

(c)
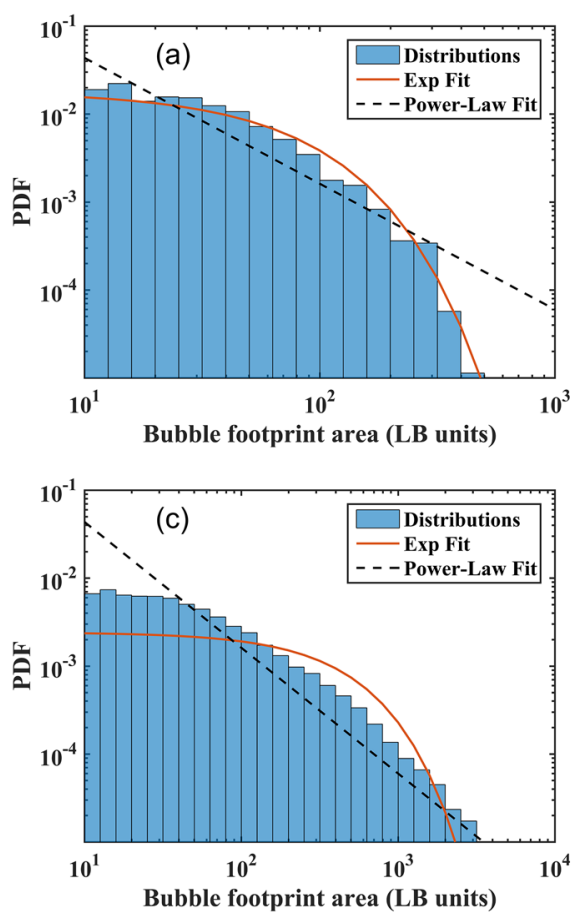

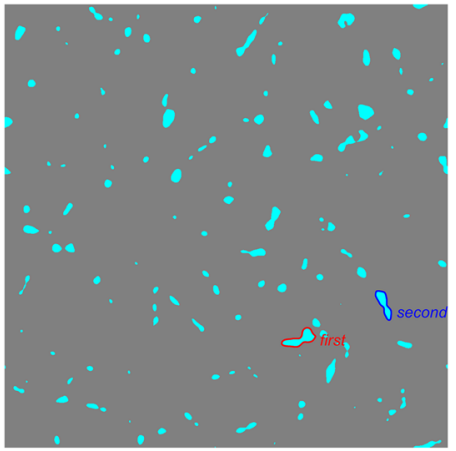

(b)

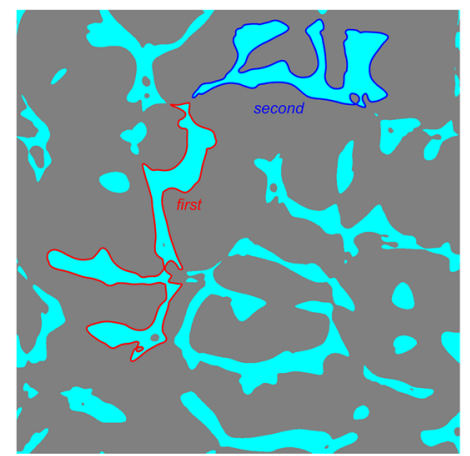

(d)
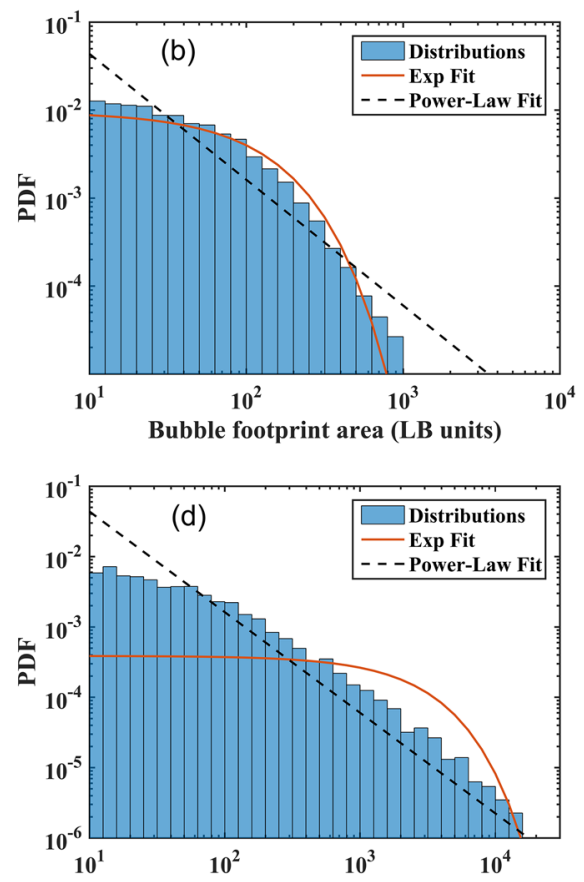

Bubble footprint area (LB units)
FIG. 13. (a) A snapshot for the pool boiling process at $\mathrm{Ja}=0.220$. The red rectangle shows the location of the slice. Typical bubble footprint distributions at $\mathrm{Ja}=0.220,0.0283$, and 0.441 are shown in panels $(b)-(d)$, respectively.
FIG. 14. PDFs of the bubble footprint area. The solid lines and dotted lines represent the exponential fitting by Eq. (30) and the power-law fitting by Eq. (31), respectively. (a) $\mathrm{Ja}=0.189$, (b) $\mathrm{Ja}$ $=0.220$, (c) $\mathrm{Ja}=0.283$, and (d) $\mathrm{Ja}$ $=0.346$. 

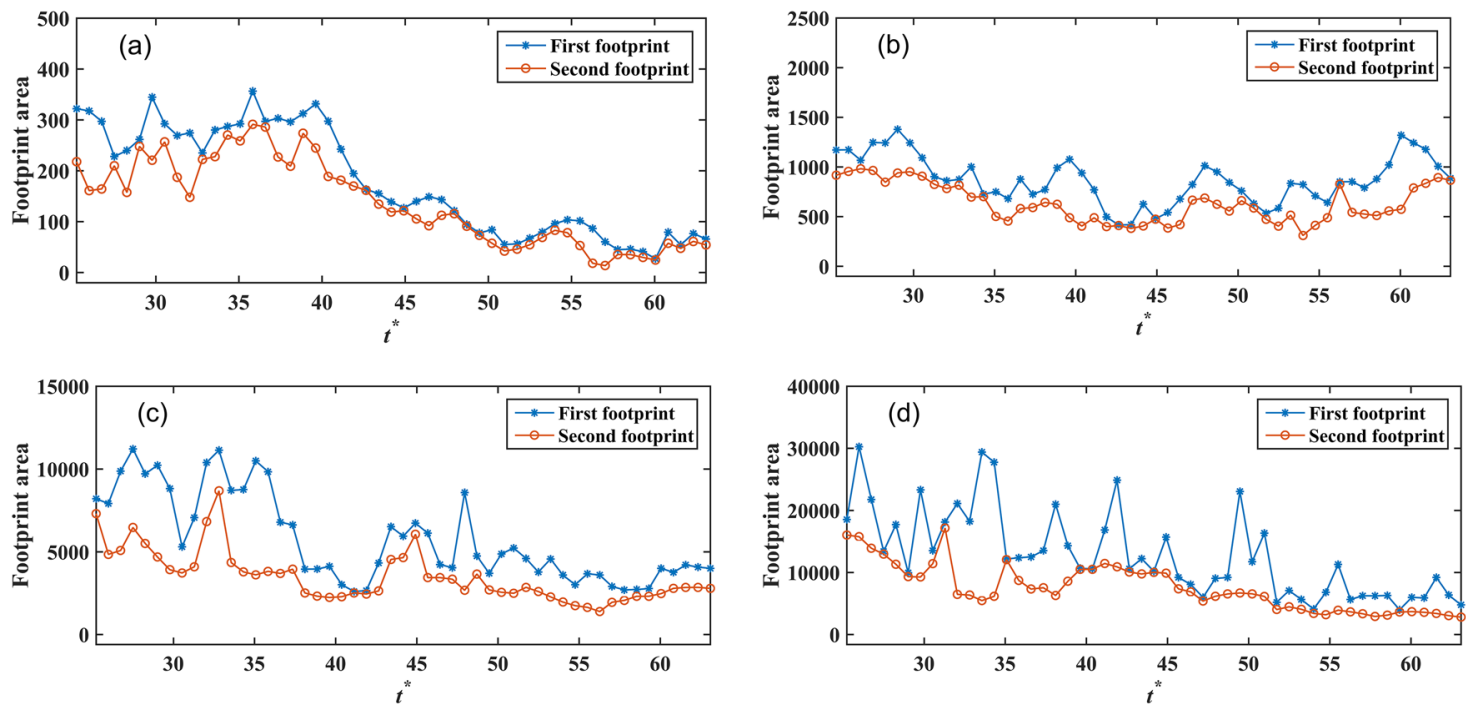

FIG. 15. Transient variation for the areas of the first two largest bubble footprints. (a) $\mathrm{Ja}=0.189$, (b) $\mathrm{Ja}=0.220$, (c) $\mathrm{Ja}=0.283$, and (d) $\mathrm{Ja}=0.346$.

increased, bubbles merge more and more frequently, and large bubble patches may appear on the wall surface. As a result, the tail of the PDF is stretched, and the PDF deviates from the exponential distribution, as presented in Figs. 14(c) and 14(d). Recent experiments showed that when the boiling crisis occurs $\left(J a \geq J a_{\mathrm{CHC}}\right)$, the PDF could be approximately fitted by a power-law distribution, i.e., $P(S)$ $=1 / S^{n}$. Based on the present 3D simulations, we could further test against such an argument. When the superheat is increased to be $J a=0.346$, the PDF indeed tends to a power-law distribution, as marked by the dotted line in Fig. 14. In addition, the $x$ coordinate starts at $10\left(\delta x^{2}\right)$, and the dotted lines in Fig. 14 are written by

$$
P(S)=\beta_{2} 1 / S^{n},
$$

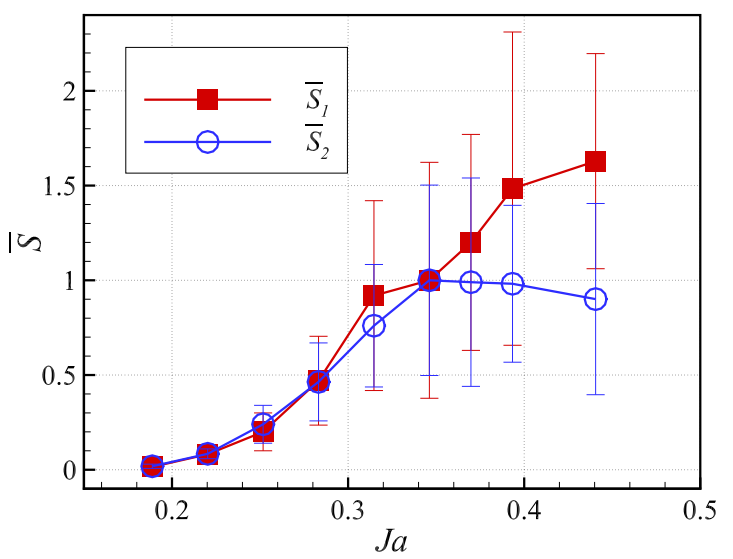

FIG. 16. Normalized average sizes for the first two largest footprints $\bar{S}_{1}$ and $\bar{S}_{2}$ change with Ja. where $\beta_{2}=1 /\left(\int_{10}^{L^{2}} 1 / S^{n} d S\right)$ is the normalization factor. In the fitting lines, the index is $n=1.43$, which is close to the index based on the experiments, $n=1.52$. $^{3}$

Figure 15 shows the transient variation for the areas of the first two largest footprints, which also represents the change in the sizes of the first two largest bubbles. As can be seen from Fig. 15, the sizes of the first two largest bubbles fluctuate significantly with time. For the small superheat case $(J a=0.189)$, despite the fluctuations, the trends of the two lines approximately overlap, as shown in Fig. 15(a). As the superheat increases, the two lines gradually bifurcate. Therefore, the dependence on the superheat may be different for the first two largest bubbles.

To show this effect, the normalized average footprint sizes (average in time and then normalized by its value at the CHF point) for the first two largest bubbles, $\bar{S}_{1}$ and $\bar{S}_{2}$, varying with $J a$, are plotted in Fig. 16. From Fig. 16, it is seen that with the increase in $J a, \bar{S}_{1}$ is increasing monotonically, while $\bar{S}_{2}$ increases first and then goes down. Such a phenomenon is reminiscent of the previous analysis that at small $J a$, the bubbles are mainly isolated (without significant coalescence among bubbles), and therefore, the first two largest bubbles do not have a large difference in average sizes. However, at large $J a$, coalescence happens frequently, and it is likely that the largest bubble engulfs the second one to form a "new and larger" largest bubble. As a result, the increase in the average size in the second largest bubble with $J a$ could be effectively suppressed. More importantly, the maximum point of $\bar{S}_{2}$ is exactly located at the CHF point, which is consistent with the experimental finding by Zhang et $a .^{3}$ and may provide a new criterion to predict the boiling crisis.

\section{CONCLUSIONS}

In this paper, a hybrid CLBM is proposed for the simulation of $3 \mathrm{D}$ thermal multiphase flow. In this model, the 
multiphase flow and temperature fields are solved by using an improved CLBM coupled with the pseudopotential model and the finite difference method, respectively, and the two fields are coupled via the non-ideal equation of state. Based on some benchmark cases, it is demonstrated that the proposed phase-change CLBM is able to simulate both the isothermal multiphase flow and the thermal multiphase flow together with liquid-vapor phase change.

The developed phase-change CLBM is then deployed to conduct high-resolution simulations of the $3 \mathrm{D}$ pool boiling process under a variety of wall superheat. The complete boiling curve with typical boiling regimes, i.e., nucleate boiling (discrete bubble region and mushroom bubble region), transition boiling, and film boiling regimes, is successfully reproduced, and the complex bubble dynamics including bubble nucleation, growth, departure, and coalescence are well captured. It is shown that the CHF predicted by the present $3 \mathrm{D}$ simulations agrees better with the existing theories and correlation models than that obtained by $2 \mathrm{D}$ simulations. Based on the large datasets with hundreds of bubbles of different sizes, a detailed analysis of the bubble footprints is then presented. It is found that the PDF of the bubble footprint area changes from an exponential distribution to a power-law distribution with the increase in the wall superheat (or Jacob number, $\mathrm{Ja}$ ), which confirms the recent experimental finding of Zhang et $a .^{3}$ Moreover, the sizes of the first two largest footprints fluctuate significantly with time. On the other hand, their normalized average sizes $\left(\bar{S}_{1}\right.$ and $\left.\bar{S}_{2}\right)$ change with $J a$ in well defined patterns. Namely, with the increase in $J a, \bar{S}_{1}$ increases monotonically, while $\bar{S}_{2}$ goes up first and then goes down, with the maximum point exactly located at the CHF point. These findings provide fresh insights into the dynamics and mechanisms of the pool boiling phenomenon.

It should be noted that the present simulations use a much lower density ratio than the one in the experiment $(\sim 1000)$. The simulations at the actual density ratio will be challenging as significantly higher computing resources are needed for high-resolution 3D timeresolved simulations. Moreover, as the density ratio increases, the increased spurious velocities at the phase interfaces would usually lead to numerical inaccuracy and/or instability. In the future, it would be informative to compare the present results with $3 \mathrm{D}$ boiling simulations at realistic density ratios. The capability of CLBM for simulating isothermal multiphase flows with high density ratios $(\gg 1000)$ and low spurious velocities has been demonstrated in Ref. 17, and the extension to boiling simulations would be another step forward.

\section{ACKNOWLEDGMENTS}

Support from the MOST National Key Research and Development Programme (Project No. 2016YFB0600805) and the Center for Combustion Energy at Tsinghua University is gratefully acknowledged. The simulations were partly performed on the Tsinghua High-Performance Parallel Computer supported by the Tsinghua National Laboratory for Information Science and Technology and partly on ARCHER funded under the EPSRC project "UK Consortium on Mesoscale Engineering Sciences (UKCOMES)" (Grant Nos. $\mathrm{EP} / \mathrm{R} 029598 / 1$ and EP/P022243/1).

\section{APPENDIX A: TRANSFORMATION MATRIX AND SHIFT MATRIX}

According to the definition in Eq. (4) of the main text, the explicit expression for $\mathbf{M}$ can be given by ${ }^{45}$

$$
\mathbf{M}=\left[\begin{array}{ccccccccccccccccccc}
1 & 1 & 1 & 1 & 1 & 1 & 1 & 1 & 1 & 1 & 1 & 1 & 1 & 1 & 1 & 1 & 1 & 1 & 1 \\
0 & 1 & -1 & 0 & 0 & 0 & 0 & 1 & -1 & 1 & -1 & 1 & -1 & 1 & -1 & 0 & 0 & 0 & 0 \\
0 & 0 & 0 & 1 & -1 & 0 & 0 & 1 & 1 & -1 & -1 & 0 & 0 & 0 & 0 & 1 & -1 & 1 & -1 \\
0 & 0 & 0 & 0 & 0 & 1 & -1 & 0 & 0 & 0 & 0 & 1 & 1 & -1 & -1 & 1 & 1 & -1 & -1 \\
0 & 0 & 0 & 0 & 0 & 0 & 0 & 1 & -1 & -1 & 1 & 0 & 0 & 0 & 0 & 0 & 0 & 0 & 0 \\
0 & 0 & 0 & 0 & 0 & 0 & 0 & 0 & 0 & 0 & 0 & 1 & -1 & -1 & 1 & 0 & 0 & 0 & 0 \\
0 & 0 & 0 & 0 & 0 & 0 & 0 & 0 & 0 & 0 & 0 & 0 & 0 & 0 & 0 & 1 & -1 & -1 & 1 \\
0 & 1 & 1 & 0 & 0 & 0 & 0 & 1 & 1 & 1 & 1 & 1 & 1 & 1 & 1 & 0 & 0 & 0 & 0 \\
0 & 0 & 0 & 1 & 1 & 0 & 0 & 1 & 1 & 1 & 1 & 0 & 0 & 0 & 0 & 1 & 1 & 1 & 1 \\
0 & 0 & 0 & 0 & 0 & 1 & 1 & 0 & 0 & 0 & 0 & 1 & 1 & 1 & 1 & 1 & 1 & 1 & 1 \\
0 & 0 & 0 & 0 & 0 & 0 & 0 & 1 & -1 & 1 & -1 & 0 & 0 & 0 & 0 & 0 & 0 & 0 & 0 \\
0 & 0 & 0 & 0 & 0 & 0 & 0 & 0 & 0 & 0 & 0 & 1 & -1 & 1 & -1 & 0 & 0 & 0 & 0 \\
0 & 0 & 0 & 0 & 0 & 0 & 0 & 1 & 1 & -1 & -1 & 0 & 0 & 0 & 0 & 0 & 0 & 0 & 0 \\
0 & 0 & 0 & 0 & 0 & 0 & 0 & 0 & 0 & 0 & 0 & 1 & 1 & -1 & -1 & 0 & 0 & 0 & 0 \\
0 & 0 & 0 & 0 & 0 & 0 & 0 & 0 & 0 & 0 & 0 & 0 & 0 & 0 & 0 & 1 & -1 & 1 & -1 \\
0 & 0 & 0 & 0 & 0 & 0 & 0 & 0 & 0 & 0 & 0 & 0 & 0 & 0 & 0 & 1 & 1 & -1 & -1 \\
0 & 0 & 0 & 0 & 0 & 0 & 0 & 1 & 1 & 1 & 1 & 0 & 0 & 0 & 0 & 0 & 0 & 0 & 0 \\
0 & 0 & 0 & 0 & 0 & 0 & 0 & 0 & 0 & 0 & 0 & 1 & 1 & 1 & 1 & 0 & 0 & 0 & 0 \\
0 & 0 & 0 & 0 & 0 & 0 & 0 & 0 & 0 & 0 & 0 & 0 & 0 & 0 & 0 & 1 & 1 & 1 & 1
\end{array}\right] .
$$


It can be seen that $\mathbf{M}$ is a non-orthogonal matrix. Its inverse is also a non-orthogonal matrix, which can be obtained by the software MATLAB, namely,

$$
\mathbf{M}^{-1}=\frac{1}{4}\left[\begin{array}{ccccccccccccccccccc}
4 & 0 & 0 & 0 & 0 & 0 & 0 & -4 & -4 & -4 & 0 & 0 & 0 & 0 & 0 & 0 & 4 & 4 & 4 \\
0 & 2 & 0 & 0 & 0 & 0 & 0 & 2 & 0 & 0 & -2 & -2 & 0 & 0 & 0 & 0 & -2 & -2 & 0 \\
0 & -2 & 0 & 0 & 0 & 0 & 0 & 2 & 0 & 0 & 2 & 2 & 0 & 0 & 0 & 0 & -2 & -2 & 0 \\
0 & 0 & 2 & 0 & 0 & 0 & 0 & 0 & 2 & 0 & 0 & 0 & -2 & 0 & -2 & 0 & -2 & 0 & -2 \\
0 & 0 & -2 & 0 & 0 & 0 & 0 & 0 & 2 & 0 & 0 & 0 & 2 & 0 & 2 & 0 & -2 & 0 & -2 \\
0 & 0 & 0 & 2 & 0 & 0 & 0 & 0 & 0 & 2 & 0 & 0 & 0 & -2 & 0 & -2 & 0 & -2 & -2 \\
0 & 0 & 0 & -2 & 0 & 0 & 0 & 0 & 0 & 2 & 0 & 0 & 0 & 2 & 0 & 2 & 0 & -2 & -2 \\
0 & 0 & 0 & 0 & 1 & 0 & 0 & 0 & 0 & 0 & 1 & 0 & 1 & 0 & 0 & 0 & 1 & 0 & 0 \\
0 & 0 & 0 & 0 & -1 & 0 & 0 & 0 & 0 & 0 & -1 & 0 & 1 & 0 & 0 & 0 & 1 & 0 & 0 \\
0 & 0 & 0 & 0 & -1 & 0 & 0 & 0 & 0 & 0 & 1 & 0 & -1 & 0 & 0 & 0 & 1 & 0 & 0 \\
0 & 0 & 0 & 0 & 1 & 0 & 0 & 0 & 0 & 0 & -1 & 0 & -1 & 0 & 0 & 0 & 1 & 0 & 0 \\
0 & 0 & 0 & 0 & 0 & 1 & 0 & 0 & 0 & 0 & 0 & 1 & 0 & 1 & 0 & 0 & 0 & 1 & 0 \\
0 & 0 & 0 & 0 & 0 & -1 & 0 & 0 & 0 & 0 & 0 & -1 & 0 & 1 & 0 & 0 & 0 & 1 & 0 \\
0 & 0 & 0 & 0 & 0 & -1 & 0 & 0 & 0 & 0 & 0 & 1 & 0 & -1 & 0 & 0 & 0 & 1 & 0 \\
0 & 0 & 0 & 0 & 0 & 1 & 0 & 0 & 0 & 0 & 0 & -1 & 0 & -1 & 0 & 0 & 0 & 1 & 0 \\
0 & 0 & 0 & 0 & 0 & 0 & 1 & 0 & 0 & 0 & 0 & 0 & 0 & 0 & 1 & 1 & 0 & 0 & 1 \\
0 & 0 & 0 & 0 & 0 & 0 & -1 & 0 & 0 & 0 & 0 & 0 & 0 & 0 & -1 & 1 & 0 & 0 & 1 \\
0 & 0 & 0 & 0 & 0 & 0 & -1 & 0 & 0 & 0 & 0 & 0 & 0 & 0 & 1 & -1 & 0 & 0 & 1 \\
0 & 0 & 0 & 0 & 0 & 0 & 1 & 0 & 0 & 0 & 0 & 0 & 0 & 0 & -1 & -1 & 0 & 0 & 1
\end{array}\right] .
$$

According to the relation between the raw and central moments, it can be proved that the shirt matrix $\mathbf{N}$ is a lower triangular matrix. Moreover, the inverse of the shift matrix $\mathbf{N}^{-1}$ is quite similar to $\mathbf{N}$, which can be obtained by reversing the signs of all the odd order velocity terms in $\mathbf{N} .^{45}$ The explicit formulations of $\mathbf{N}$ and $\mathbf{N}^{-1}$ are given as follows:

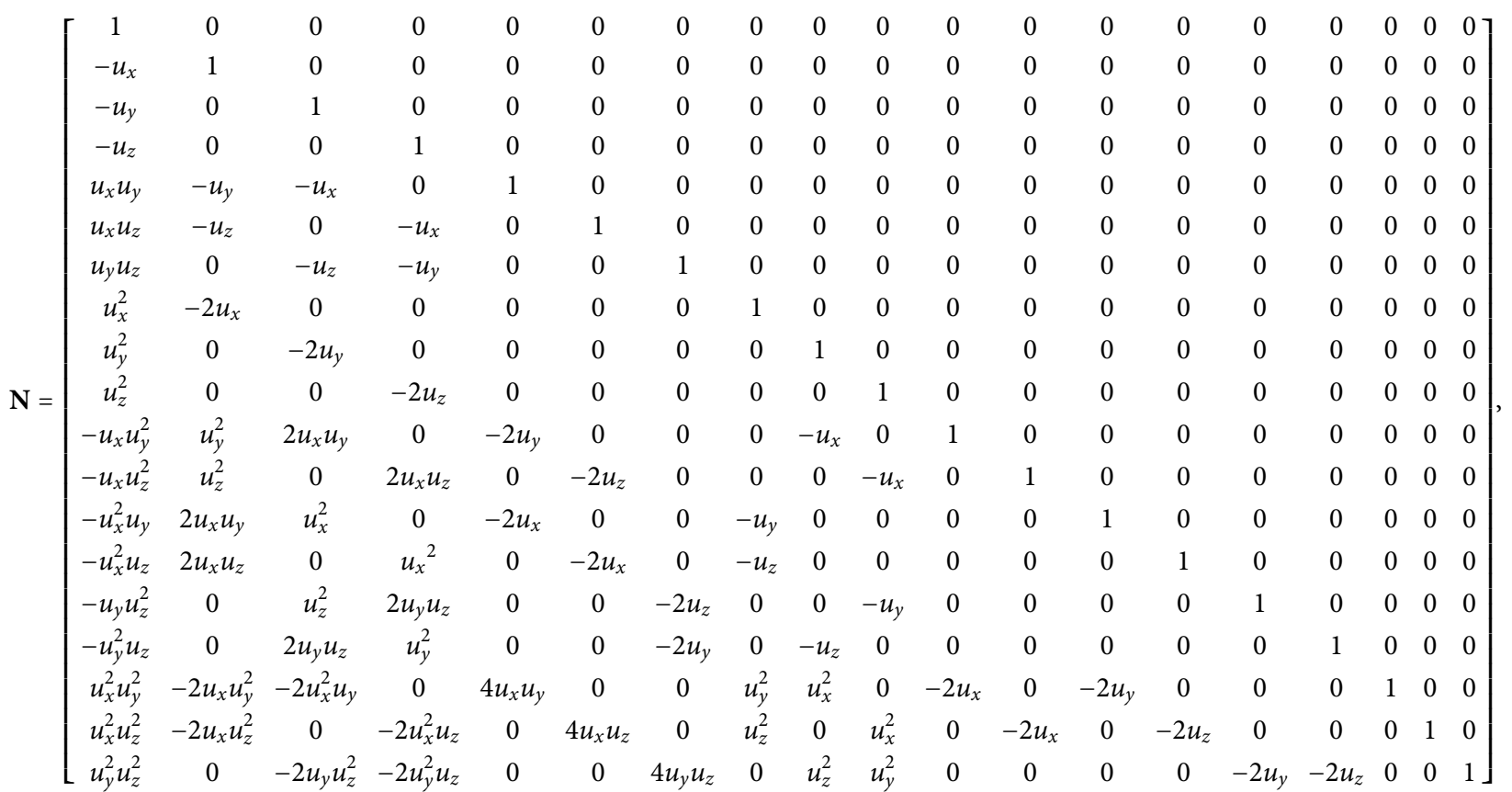




$$
\mathbf{N}^{-1}=\left[\begin{array}{ccccccccccccccccccc}
1 & 0 & 0 & 0 & 0 & 0 & 0 & 0 & 0 & 0 & 0 & 0 & 0 & 0 & 0 & 0 & 0 & 0 & 0 \\
u_{x} & 1 & 0 & 0 & 0 & 0 & 0 & 0 & 0 & 0 & 0 & 0 & 0 & 0 & 0 & 0 & 0 & 0 & 0 \\
u_{y} & 0 & 1 & 0 & 0 & 0 & 0 & 0 & 0 & 0 & 0 & 0 & 0 & 0 & 0 & 0 & 0 & 0 & 0 \\
u_{z} & 0 & 0 & 1 & 0 & 0 & 0 & 0 & 0 & 0 & 0 & 0 & 0 & 0 & 0 & 0 & 0 & 0 & 0 \\
u_{x} u_{y} & u_{y} & u_{x} & 0 & 1 & 0 & 0 & 0 & 0 & 0 & 0 & 0 & 0 & 0 & 0 & 0 & 0 & 0 & 0 \\
u_{x} u_{z} & u_{z} & 0 & u_{x} & 0 & 1 & 0 & 0 & 0 & 0 & 0 & 0 & 0 & 0 & 0 & 0 & 0 & 0 & 0 \\
u_{y} u_{z} & 0 & u_{z} & u_{y} & 0 & 0 & 1 & 0 & 0 & 0 & 0 & 0 & 0 & 0 & 0 & 0 & 0 & 0 & 0 \\
u_{x}^{2} & 2 u_{x} & 0 & 0 & 0 & 0 & 0 & 1 & 0 & 0 & 0 & 0 & 0 & 0 & 0 & 0 & 0 & 0 & 0 \\
u_{y}^{2} & 0 & 2 u_{y} & 0 & 0 & 0 & 0 & 0 & 1 & 0 & 0 & 0 & 0 & 0 & 0 & 0 & 0 & 0 & 0 \\
u_{z}^{2} & 0 & 0 & 2 u_{z} & 0 & 0 & 0 & 0 & 0 & 1 & 0 & 0 & 0 & 0 & 0 & 0 & 0 & 0 & 0 \\
u_{x} u_{y}^{2} & u_{y}^{2} & 2 u_{x} u_{y} & 0 & 2 u_{y} & 0 & 0 & 0 & u_{x} & 0 & 1 & 0 & 0 & 0 & 0 & 0 & 0 & 0 & 0 \\
u_{x} u_{z}^{2} & u_{z}^{2} & 0 & 2 u_{x} u_{z} & 0 & 2 u_{z} & 0 & 0 & 0 & u_{x} & 0 & 1 & 0 & 0 & 0 & 0 & 0 & 0 & 0 \\
u_{x}^{2} u_{y} & 2 u_{x} u_{y} & u_{x}^{2} & 0 & 2 u_{x} & 0 & 0 & u_{y} & 0 & 0 & 0 & 0 & 1 & 0 & 0 & 0 & 0 & 0 & 0 \\
u_{x}^{2} u_{z} & 2 u_{x} u_{z} & 0 & u_{x}^{2} & 0 & 2 u_{x} & 0 & u_{z} & 0 & 0 & 0 & 0 & 0 & 1 & 0 & 0 & 0 & 0 & 0 \\
u_{y} u_{z}^{2} & 0 & u_{z}^{2} & 2 u_{y} u_{z} & 0 & 0 & 2 u_{z} & 0 & 0 & u_{y} & 0 & 0 & 0 & 0 & 1 & 0 & 0 & 0 & 0 \\
u_{y}^{2} u_{z} & 0 & 2 u_{y} u_{z} & u_{y}^{2} & 0 & 0 & 2 u_{y} & 0 & u_{z} & 0 & 0 & 0 & 0 & 0 & 0 & 1 & 0 & 0 & 0 \\
u_{x}^{2} u_{y}^{2} & 2 u_{x} u_{y}^{2} & 2 u_{x}^{2} u_{y} & 0 & 4 u_{x} u_{y} & 0 & 0 & u_{y}^{2} & u_{x}^{2} & 0 & 2 u_{x} & 0 & 2 u_{y} & 0 & 0 & 0 & 1 & 0 & 0 \\
u_{x}^{2} u_{z}^{2} & 2 u_{x} u_{z}^{2} & 0 & 2 u_{x}^{2} u_{z} & 0 & 4 u_{x} u_{z} & 0 & u_{z}^{2} & 0 & u_{x}^{2} & 0 & 2 u_{x} & 0 & 2 u_{z} & 0 & 0 & 0 & 1 & 0 \\
u_{y}^{2} u_{z}^{2} & 0 & 2 u_{y} u_{z}^{2} & 2 u_{y}^{2} u_{z} & 0 & 0 & 4 u_{y} u_{z} & 0 & u_{z}^{2} & u_{y}^{2} & 0 & 0 & 0 & 0 & 2 u_{y} & 2 u_{z} & 0 & 0 & 1
\end{array}\right]
$$

\section{APPENDIX B: NON-EQUILIBRIUM BOUNCE-BACK SCHEME}

Taking the bottom wall $(z=0)$ as an example, the distribution functions along the velocity directions, $\mathbf{e}_{5}, \mathbf{e}_{11}, \mathbf{e}_{12}, \mathbf{e}_{15}$, and $\mathbf{e}_{18}$, are unknown because they are incoming from the outside of the computational domain. According to the ideology of the non-equilibrium bounce-back scheme by Zou and He, ${ }^{68}$ the unknown distribution functions can be reconstructed by

$$
\begin{aligned}
& f_{5}=f_{6}, \\
& f_{11}=f_{14}-0.5\left(-f_{2}-f_{8}-f_{10}+f_{1}+f_{7}+f_{9}\right), \\
& f_{12}=f_{13}+0.5\left(-f_{2}-f_{8}-f_{10}+f_{1}+f_{7}+f_{9}\right), \\
& f_{15}=f_{18}-0.5\left(f_{3}+f_{7}+f_{8}-f_{4}-f_{9}-f_{10}\right), \\
& f_{16}=f_{17}+0.5\left(f_{3}+f_{7}+f_{8}-f_{4}-f_{9}-f_{10}\right) .
\end{aligned}
$$

The unknown distribution functions on the top wall, $f_{6}, f_{13}, f_{14}, f_{17}$, and $f_{18}$ can be also obtained according to the above relations.

\section{DATA AVAILABILITY}

The data that support the findings of this study are available from the corresponding author upon reasonable request.

\section{REFERENCES}

'S. Nukiyama, "The maximum and minimum values of the heat $\mathrm{Q}$ transmitted from metal to boiling water under atmospheric pressure," J. Soc. Mech. Eng. 37, 367-374 (1934).

${ }^{2}$ V. K. Dhir, "Boiling heat transfer," Annu. Rev. Fluid Mech. 30, 365-401 (1998).
${ }^{3}$ L. Zhang, J. H. Seong, and M. Bucci, "Percolative scale-free behavior in the boiling crisis," Phys. Rev. Lett. 122, 134501 (2019).

${ }^{4} \mathrm{~J}$. Du, C. Zhao, and H. Bo, "Investigation of bubble departure diameter in horizontal and vertical subcooled flow boiling," Int. J. Heat Mass Transfer 127, 796-805 (2018).

${ }^{5}$ J. Du, C. Zhao, and H. Bo, "A modified model for bubble growth rate and bubble departure diameter in nucleate pool boiling covering a wide range of pressures," Appl. Therm. Eng. 145, 407-415 (2018).

${ }^{6}$ W. M. Rohsenow, "A method of correlating heat transfer data for surface boiling of liquids," Report No. 5 https://dspace.mit.edu/handle/1721.1/61431 MIT Division of Industrial Cooporation, Cambridge, Mass., 1951.

${ }^{7} \mathrm{H}$. Ohtake and Y. Koizumi, "Derivations of correlation and liquid-solid contact model of transition boiling heat transfer," JSME Int. J., Ser. B 49, 343-351 (2006).

${ }^{8}$ N. Zuber, Hydrodynamic Aspects of Boiling Heat Transfer (United States Atomic Energy Commission, Technical Information Service, 1959), p. 4439.

${ }^{9} \mathrm{X}$. Xu and T. Qian, "Single-bubble dynamics in pool boiling of one-component fluids," Phys. Rev. E 89, 063002 (2014).

${ }^{10} \mathrm{G}$. Son and V. K. Dhir, "Numerical simulation of saturated film boiling on a horizontal surface," J. Heat Transfer 119, 525 (1997).

${ }^{11}$ D. Juric and G. Tryggvason, “Computations of boiling flows," Int. J. Multiphase Flow 24, 387-410 (1998).

${ }^{12} \mathrm{~S}$. W. J. Welch and J. Wilson, "A volume of fluid based method for fluid flows with phase change," J. Comput. Phys. 160, 662-682 (2000).

${ }^{13} \mathrm{~T}$. Kunugi, "Brief review of latest direct numerical simulation on pool and film boiling," Nucl. Eng. Technol. 44, 847-854 (2012).

${ }^{14}$ V. K. Dhir, G. R. Warrier, and E. Aktinol, "Numerical simulation of pool boiling: A review," J. Heat Transfer 135, 061502 (2013).

${ }^{15} \mathrm{X}$. Shan and H. Chen, "Lattice Boltzmann model for simulating flows with multiple phases and components," Phys. Rev. E 47, 1815 (1993).

${ }^{16}$ Y. Gan, A. Xu, G. Zhang, and S. Succi, "Discrete Boltzmann modeling of multiphase flows: Hydrodynamic and thermodynamic non-equilibrium effects," Soft Matter 11, 5336-5345 (2015). 
${ }^{17}$ D. Lycett-Brown and K. H. Luo, "Cascaded lattice Boltzmann method with improved forcing scheme for large-density-ratio multiphase flow at high Reynolds and Weber numbers," Phys. Rev. E 94, 053313 (2016).

${ }^{18}$ Q. Li, K. H. Luo, Q. J. Kang, Y. L. He, Q. Chen, and Q. Liu, "Lattice Boltzmann methods for multiphase flow and phase-change heat transfer," Prog. Energy Combust. Sci. 52, 62-105 (2016).

${ }^{19}$ H. Liu, Q. Kang, C. R. Leonardi, S. Schmieschek, A. Narváez, B. D. Jones, J. R. Williams, A. J. Valocchi, and J. Harting, "Multiphase lattice Boltzmann simulations for porous media applications," Comput. Geosci. 20, 777-805 (2016).

${ }^{20}$ L. Fei, A. Scagliarini, A. Montessori, M. Lauricella, S. Succi, and K. H. Luo, "Mesoscopic model for soft flowing systems with tunable viscosity ratio," Phys. Rev. Fluids 3, 104304 (2018).

${ }^{21}$ S. Saito, A. De Rosis, A. Festuccia, A. Kaneko, Y. Abe, and K. Koyama, "Color-gradient lattice Boltzmann model with nonorthogonal central moments: Hydrodynamic melt-jet breakup simulations," Phys. Rev. E 98, 013305 (2018).

${ }^{22}$ F. Qin, A. Mazloomi Moqaddam, Q. Kang, D. Derome, and J. Carmeliet, "Entropic multiple-relaxation-time multirange pseudopotential lattice Boltzmann model for two-phase flow," Phys. Fluids 30, 032104 (2018).

${ }^{23}$ L. Fei, J. Du, K. H. Luo, S. Succi, M. Lauricella, A. Montessori, and Q. Wang, "Modeling realistic multiphase flows using a non-orthogonal multiple-relaxationtime lattice Boltzmann method," Phys. Fluids 31, 042105 (2019).

${ }^{24}$ Y.-B. Gan, A.-G. Xu, G.-C. Zhang, C.-D. Lin, H.-L. Lai, and Z.-P. Liu, "Nonequilibrium and morphological characterizations of Kelvin-Helmholtz instability in compressible flows," Front. Phys. 14, 43602 (2019).

${ }^{25}$ É. Ruiz-Gutiérrez and R. Ledesma-Aguilar, "Lattice-Boltzmann simulations of electrowetting phenomena," Langmuir 35, 4849-4859 (2019).

${ }^{26}$ F. Qin, L. Del Carro, A. Mazloomi Moqaddam, Q. Kang, T. Brunschwiler, D. Derome, and J. Carmeliet, "Study of non-isothermal liquid evaporation in synthetic micro-pore structures with hybrid lattice Boltzmann model," J. Fluid Mech. 866, 33-60 (2019).

${ }^{27}$ J. Yang, X. Ma, L. Fei, X. Zhang, K. H. Luo, and S. Shuai, "Effects of hysteresis window on contact angle hysteresis behaviour at large bond number," J. Colloid Interface Sci. 566, 327-337 (2020).

${ }^{28}$ L. Fei, A. Scagliarini, K. H. Luo, and S. Succi, "Discrete fluidization of dense monodisperse emulsions in neutral wetting microchannels," Soft Matter 16, 651-658 (2020).

${ }^{29}$ Y. Yu, Q. Li, Z. X. Wen, and R. Z. Huang, "Investigation on boundary schemes in lattice Boltzmann simulations of boiling heat transfer involving curved surfaces," Phys. Fluids 32, 063305 (2020).

${ }^{30}$ Q. Li, Q. J. Kang, M. M. Francois, Y. L. He, and K. H. Luo, "Lattice Boltzmann modeling of boiling heat transfer: The boiling curve and the effects of wettability," Int. J. Heat Mass Transfer 85, 787-796 (2015).

${ }^{31}$ M. C. Sukop and D. Or, "Lattice Boltzmann method for modeling liquidvapor interface configurations in porous media," Water Resour. Res. 40, W01509, https://doi.org/10.1029/2003wr002333 (2004).

${ }^{32}$ S. Popinet, "An accurate adaptive solver for surface-tension-driven interfacial flows," J. Comput. Phys. 228, 5838-5866 (2009).

${ }^{33} \mathrm{R}$. Zhang and H. Chen, "Lattice Boltzmann method for simulations of liquidvapor thermal flows,” Phys. Rev. E 67, 066711 (2003).

${ }^{34}$ G. Házi and A. Márkus, "Modeling heat transfer in supercritical fluid using the lattice Boltzmann method," Phys. Rev. E 77, 026305 (2008).

${ }^{35}$ L. Biferale, P. Perlekar, M. Sbragaglia, and F. Toschi, "Convection in multiphase fluid flows using lattice Boltzmann methods," Phys. Rev. Lett. 108, 104502 (2012).

${ }^{36}$ S. Gong and P. Cheng, "A lattice Boltzmann method for simulation of liquidvapor phase-change heat transfer," Int. J. Heat Mass Transfer 55, 4923-4927 (2012).

${ }^{37} \mathrm{Q}$. Li and K. H. Luo, "Effect of the forcing term in the pseudopotential lattice Boltzmann modeling of thermal flows," Phys. Rev. E 89, 053022 (2014).

${ }^{38} \mathrm{Q}$. Li, Y. Yu, and Z. X. Wen, "How does boiling occur in lattice Boltzmann simulations?," Phys. Fluids 32, 093306 (2020).

${ }^{39}$ Y. H. Qian, D. d'Humieres, and P. Lallemand, "Lattice BGK models for NavierStokes equation,” Europhys. Lett. 17, 479 (1992).

${ }^{40}$ D. d'Humieres, "Generalized lattice-Boltzmann equations," Prog. Astronaut. Aeronaut. 159, 450-458 (1992).
${ }^{41}$ P. Lallemand and L.-S. Luo, "Theory of the lattice Boltzmann method: Dispersion, dissipation, isotropy, Galilean invariance, and stability," Phys. Rev. E 61, 6546 (2000).

${ }^{42}$ M. Geier, A. Greiner, and J. G. Korvink, "Cascaded digital lattice Boltzmann automata for high Reynolds number flow," Phys. Rev. E 73, 066705 (2006).

${ }^{43}$ D. Lycett-Brown and K. H. Luo, "Multiphase cascaded lattice Boltzmann method," Comput. Math. Appl. 67, 350-362 (2014).

${ }^{44} \mathrm{~L}$. Fei and K. H. Luo, "Consistent forcing scheme in the cascaded lattice Boltzmann method," Phys. Rev. E 96, 053307 (2017).

${ }^{45}$ L. Fei, K. H. Luo, and Q. Li, "Three-dimensional cascaded lattice Boltzmann method: Improved implementation and consistent forcing scheme," Phys. Rev. E 97, 053309 (2018).

${ }^{46} \mathrm{~A}$. De Rosis, "Alternative formulation to incorporate forcing terms in a lattice Boltzmann scheme with central moments," Phys. Rev. E 95, 023311 (2017).

${ }^{47}$ K. N. Premnath and S. Banerjee, "Incorporating forcing terms in cascaded lattice Boltzmann approach by method of central moments," Phys. Rev. E 80, 036702 (2009).

${ }^{48}$ L. Fei, K. H. Luo, C. Lin, and Q. Li, "Modeling incompressible thermal flows using a central-moments-based lattice Boltzmann method," Int. J. Heat Mass Transfer 120, 624-634 (2018).

${ }^{49}$ L. Fei and K. H. Luo, "Cascaded lattice Boltzmann method for incompressible thermal flows with heat sources and general thermal boundary conditions," Comput. Fluids 165, 89-95 (2018).

${ }^{50}$ S. Saito, A. De Rosis, L. Fei, K. H. Luo, K.-I. Ebihara, A. Kaneko, Y. Abe, and K. Koyama, "Lattice Boltzmann modeling and simulation of forced-convection boiling on a cylinder," arXiv:1912.02018 (2019).

${ }^{51} \mathrm{X}$. Shan and $\mathrm{H}$. Chen, "Simulation of nonideal gases and liquid-gas phase transitions by the lattice Boltzmann equation," Phys. Rev. E 49, 2941 (1994).

${ }^{52}$ P. Yuan and L. Schaefer, "Equations of state in a lattice Boltzmann model," Phys. Fluids 18, 042101 (2006).

${ }^{53} \mathrm{Q}$. Li, K. H. Luo, X. Li et al., "Forcing scheme in pseudopotential lattice Boltzmann model for multiphase flows," Phys. Rev. E 86, 016709 (2012).

${ }^{54} \mathrm{Q}$. Li, K. H. Luo, and X. Li, "Lattice Boltzmann modeling of multiphase flows at large density ratio with an improved pseudopotential model," Phys. Rev. E 87, 053301 (2013).

${ }^{55}$ T. Lee and C.-L. Lin, "A stable discretization of the lattice Boltzmann equation for simulation of incompressible two-phase flows at high density ratio," J. Comput. Phys. 206, 16-47 (2005).

${ }^{56}$ H. Huang, D. T. Thorne, Jr., M. G. Schaap, and M. C. Sukop, "Proposed approximation for contact angles in Shan-and-Chen-type multicomponent multiphase lattice Boltzmann models," Phys. Rev. E 76, 066701 (2007).

${ }^{57}$ C. K. Law, "Recent advances in droplet vaporization and combustion," Prog. Energy Combust. Sci. 8, 171-201 (1982).

${ }^{58}$ Q. Li, K. H. Luo, Q. Kang, and Q. Chen, "Contact angles in the pseudopotential lattice Boltzmann modeling of wetting," Phys. Rev. E 90, 053301 (2014).

${ }^{59}$ G. Son, V. K. Dhir, and N. Ramanujapu, "Dynamics and heat transfer associated with a single bubble during nucleate boiling on a horizontal surface," J. Heat Transfer 121, 623-631 (1999)

${ }^{60} \mathrm{~J}$. Latt, Choice of units in lattice Boltzmann simulations, available at: http://lbmethod.org/_media/howtos:Ibunits.pdf, 2008.

${ }^{61}$ N. R. Koosukuntla, "Towards development of a multiphase simulation model using lattice Boltzmann method (LBM)," Ph.D. thesis, University of Toledo, 2011.

${ }^{62}$ S. Gong and P. Cheng, "Lattice Boltzmann simulation of periodic bubble nucleation, growth and departure from a heated surface in pool boiling," Int. J. Heat Mass Transfer 64, 122-132 (2013).

${ }^{63}$ Y. H. Chang and Y. M. Ferng, "Experimental investigation on bubble dynamics and boiling heat transfer for saturated pool boiling and comparison data with previous works," Appl. Therm. Eng. 154, 284-293 (2019).

${ }^{64}$ G. Kocamustafaogullari and M. Ishii, "Interfacial area and nucleation site density in boiling systems," Int. J. Heat Mass Transfer 26, 1377-1387 (1983). 
${ }^{65}$ S. G. Kandlikar, "A theoretical model to predict pool boiling CHF incorporating effects of contact angle and orientation," J. Heat Transfer 123, 1071-1079 (2001). ${ }^{66} \mathrm{~S}$. Gong and P. Cheng, "Direct numerical simulations of pool boiling curves including heater's thermal responses and the effect of vapor phase's thermal conductivity," Int. Commun. Heat Mass Transfer 87, 61-71 (2017).
${ }^{67}$ T. Charignon, P. Lloveras, D. Chatain, L. Truskinovsky, E. Vives, D. Beysens, and V. Nikolayev, "Criticality in the slowed-down boiling crisis at zero gravity," Phys. Rev. E 91, 053007 (2015).

${ }^{68} \mathrm{Q}$. Zou and X. He, "On pressure and velocity boundary conditions for the lattice Boltzmann BGK model,” Phys. Fluids 9, 1591-1598 (1997). 